\title{
Eyecup scope-optical recordings of light stimulus-evoked fluorescence signals in the retina
}

\author{
Thomas Euler • Susanne E. Hausselt • \\ David J. Margolis • Tobias Breuninger • Xavier Castell • \\ Peter B. Detwiler • Winfried Denk
}

Received: 14 July 2008 /Revised: 9 October 2008 / Accepted: 15 October 2008 /Published online: 21 November 2008

(C) The Author(s) 2008. This article is published with open access at Springerlink.com

\begin{abstract}
Dendritic signals play an essential role in processing visual information in the retina. To study them in neurites too small for electrical recording, we developed an instrument that combines a multi-photon (MP) microscope with a through-the-objective high-resolution visual stimulator. An upright microscope was designed that uses the objective lens for both MP imaging and delivery of visual stimuli to functionally intact retinal explants or eyecup preparations. The stimulator consists of a miniature liquid-crystal-on-silicon display coupled into the optical path of an infrared-excitation laser-scanning microscope. A pair of custom-made dichroic filters allows light from the excitation laser and three spectral bands ('colors') from the stimulator to reach the retina, leaving two intermediate bands for fluorescence imaging. Special optics allow displacement of the stimulator focus relative to the imaging focus. Spatially resolved changes in calcium-indicator fluorescence in response to visual stimuli were recorded in dendrites of different types of mammalian retinal neurons.
\end{abstract}

Electronic supplementary material The online version of this article (doi:10.1007/s00424-008-0603-5) contains supplementary material, which is available to authorized users.

T. Euler $(\bowtie) \cdot$ S. E. Hausselt $\cdot$ T. Breuninger $\cdot$ X. Castell $\cdot$

W. Denk

Department of Biomedical Optics,

Max-Planck-Institute for Medical Research,

Jahnstr. 29,

69120 Heidelberg, Germany

e-mail: thomas.euler@mpimf-heidelberg.mpg.de

D. J. Margolis • P. B. Detwiler

Physiology and Biophysics,

Department Physiology \& Biophysics, University of Washington,

Seattle, WA 98195, USA
Keywords Two-photon microscopy - Optical recordings · Visual stimulation $\cdot$ Calcium imaging $\cdot$ Retinal signal processing $\cdot$ Dendritic processing

\section{Introduction}

Recent advances in microscopy have greatly increased understanding of biological mechanisms. Neurobiology, in particular, has exploited several new optical approaches to study neuronal signaling events in ways (e.g., in vivo brain imaging, [1]), at length scales (e.g., dendritic arbors and spines), and resolutions (down to single molecules) that are not possible using any other technique. Since the vast majority of these new approaches are fluorescence-based and use visible light to excite a fluorescent probe or indicator, they have not been used to their full advantage in retina studies. Using light to investigate the retina (Fig. 1a) runs into the familiar quasi-Heisenbergian dilemma where the act of observing changes the observed.

The problem with making fluorescence-based measurements in the functionally intact retina is that the light used to generate fluorescence also stimulates the retina. The wavelengths of the light used to excite the fluorescence of currently available probes by one-photon (1P) absorption range roughly from $350-700 \mathrm{~nm}$. This band of wavelengths is also efficiently absorbed by retinal photopigments and, thus, strongly excites rod and cone photoreceptors. As a result, the light used to produce fluorescence also leads to a saturating light response in the photoreceptors and effectively blinds the retina to visual stimulation for the duration of the measurement (and possibly beyond). One way to minimize this problem is to excite fluorescence using energy from the simultaneous absorption of two longwavelength photons rather than one photon at half the 

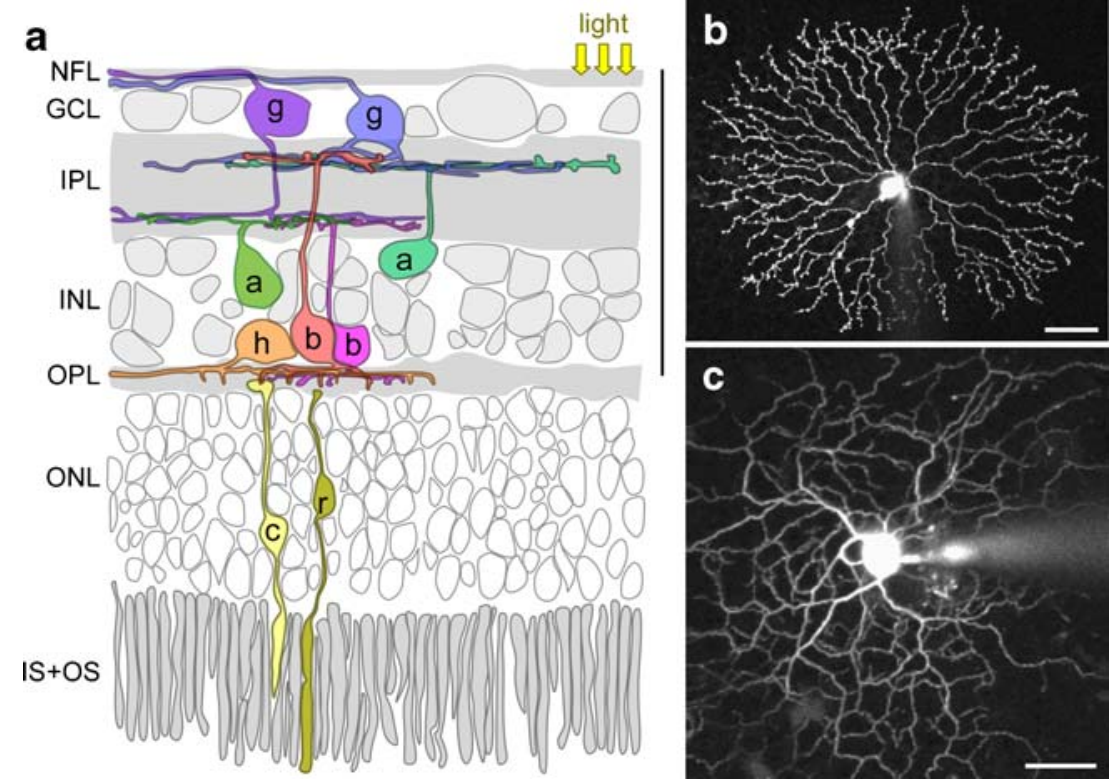

Fig. 1 Retinal cross-section and representative fluorescence images of two cell types. a Drawing of a retinal cross-section illustrating layering (NFL nerve fiber layer, GCL ganglion cell layer, IPL inner plexiform layer, $I N L$ inner nuclear layer, $O P L$ outer plexiform layer, $O N L$ outer nuclear layer, $I S+O S$ inner and outer segments) and main cell classes ( $g$ ganglion cells, $a$ amacrine cells, $h$ horizontal cell, $b$ bipolar cells, $c$ and $r$ cone and rod photoreceptor, respectively). b, c Examples of retinal neurons in flat-mounted retina filled with OGB-1 (collapsed image stacks; 'shadows' of recording electrodes are visible): a starburst amacrine cell (b) and a direction-selective ON/OFF ganglion cell (c). Scale bars: a-c $50 \mu \mathrm{m}$ wavelength. Since the long wavelength $(>900 \mathrm{~nm})$ light used for two-photon (2P) excitation of standard indicators is very inefficiently absorbed by vertebrate photoreceptors, it is possible to use $2 \mathrm{P}$, or more general, multi-photon (MP) excitation with fluorescence-based tools to study retinal neurons and their response to visual stimulation in the functionally intact retina.

The retina is a light-sensitive neural computer (reviewed in [2-5]). In vertebrates, it is made of five major cell classes, which are organized in well-defined layers mainly containing either somata (nuclear layers) or synaptic neuropil (plexiform layers) as shown in Fig. 1a. A mosaic of rod and cone photoreceptors forms the outermost cellular layer facing the back of the eye. The photoreceptors convert light into a change in membrane potential, which is then relayed synaptically to other cells in the retina. As the lightevoked signal percolates through the layers of the retinal neuronal network, salient information about intensity, chromaticity, and spatio-temporal properties of the visual scene are extracted and ultimately passed on to the retinal output neurons, the retinal ganglion cells (RGCs), which have their somata located in the innermost layer. RGCs encode the information in action potential-trains that reach higher visual centers in the brain via the optic nerve. The main goal of vision research is to understand the biophysical and network mechanisms that give rise to the neural computations needed to process visual information.
The strategies used by the retina to perform this task are undoubtedly relevant to a wide range of fundamental questions about cellular physiology and computational mechanisms in other parts of the nervous system.

Retinal neurons typically have profuse dendritic arbors (Figs 1b,c), which are a main determinant of its physiology (reviewed, e.g., in [6, 7]). Dendrites can generate responses and synaptic output independent of the soma. For example, each of the principal dendritic lobes of the starburst amacrine cell (SAC, Fig. 1b) acts as an autonomous subcellular sensor that detects the direction of image motion by generating local direction-selective $\mathrm{Ca}^{2+}$ signals $[8,9]$. In fact, most amacrine cells - the most diverse class of retinal interneurons (reviewed in [10]) - use their dendrites to receive input as well as to make output synapses. Thus, to understand the mechanisms that retinal cells use to process visual information, it is not enough to simply record the activity in the soma; it is also necessary to monitor what is happening in the dendrites. Typically, the dendrites of retinal neurons are too small to be accessed with electrodes for direct electrical recording, but they can be studied optically using fluorescent probes to monitor dendritic events.

To study neuronal signaling in the functionally intact, lightsensitive retina, we developed an instrument that combines a two-photon laser-scanning fluorescence microscope (2P microscope, [11]) with a fast (up to $300 \mathrm{~Hz}$ ) liquid crystal display (LCD) visual-stimulus generator. The instrument can 
be used with fluorescent probes that are introduced into retinal neurons by various means (e.g., via microelectrodes, by bulk-loading, or by transgene expression) to monitor intracellular signals that are generated in selected subcellular regions in response to visual stimulation.

The instrument was specifically designed to allow the microscope objective that is used for $2 \mathrm{P}$ fluorescence imaging to be used, at the same time, to deliver visual stimuli to the retina. The purpose of this particular configuration is to deliver visual stimuli through the inner surface (ganglion-cell side) of the retina; the same side that light is presented to in the intact eye. An advantage of this approach is that it allows the retina to be studied without disturbing its in situ association with the supporting epithelia that cover the outer surface (photoreceptor side) of the retina and are required for the long-term survival of retinal function. The opaque layers of supporting cells include the retina pigmented epithelium (RPE) that is the site of photopigment regeneration necessary for the maintenance of light sensitivity, the vascularized choroid, which works with the RPE to nourish the retina, and the sclera, the protective outer (white) coat of the eye that provides structural support. The in situ retina in its normal association with the supporting epithelia is referred to as the eyecup preparation, hence the name for our instrument, Eyecup scope.

\section{The Eyecup scope}

A schematic diagram of the Eyecup scope is shown in Fig. 2. It is basically an episcopic microscope that uses a single infinity-corrected microscope objective to deliver both infrared laser pulses for MP fluorescence excitation and light patterns for visual stimulation of the retina. A commercially available microscope version based on the Eyecup scope design is available from Sutter Instruments (Novato, CA, USA).

The Eyecup scope consists of two separate arms: the laser-scanning arm and the visual-stimulator arm. The optical elements that these two arms have in common include the scan lens (the equivalent of an "eye piece"), the tube lens, and the microscope objective. In addition, there are two beam-turning mirrors in the infinity space between tube lens and objective.

The purpose of the laser-scanning arm is to move a focused spot of laser light across the preparation. During scanning, the excitation laser beam pivots around a point in
Fig. 2 Schematic overview of the Eyecup scope. Drawing illustrating the different sections of the Eyecup scope; indicated are the optical paths for laser excitation light (entering from the top right; the source, a mode-locked Ti/Sapphire laser, is not shown) and the fluorescence light (emitted from the tissue in the chamber, bottom left). For details on the path of the stimulus light, see text and Fig. 5. $L E D$ light-emitting diode, $L E D_{S}$ short-wavelength, $\lambda_{\text {peak }}=400 \mathrm{~nm}, L E D_{M}$, middlewavelength, $\lambda_{\text {peak }}=570 \mathrm{~nm}$, $P M T$ photo-multiplier tube, $D M$ dichroic mirror [for specifications of $\mathrm{DM}_{\mathrm{Obj}}(\mathrm{M} 5), \mathrm{DM}_{\mathrm{vStim}}$ (M7) and $\mathrm{DM}_{\mathrm{Cam}}$ (M8) see text], $L C O S$ liquid crystal on silicon, $I R$ infrared, $B P$ band pass, $L P$ long pass

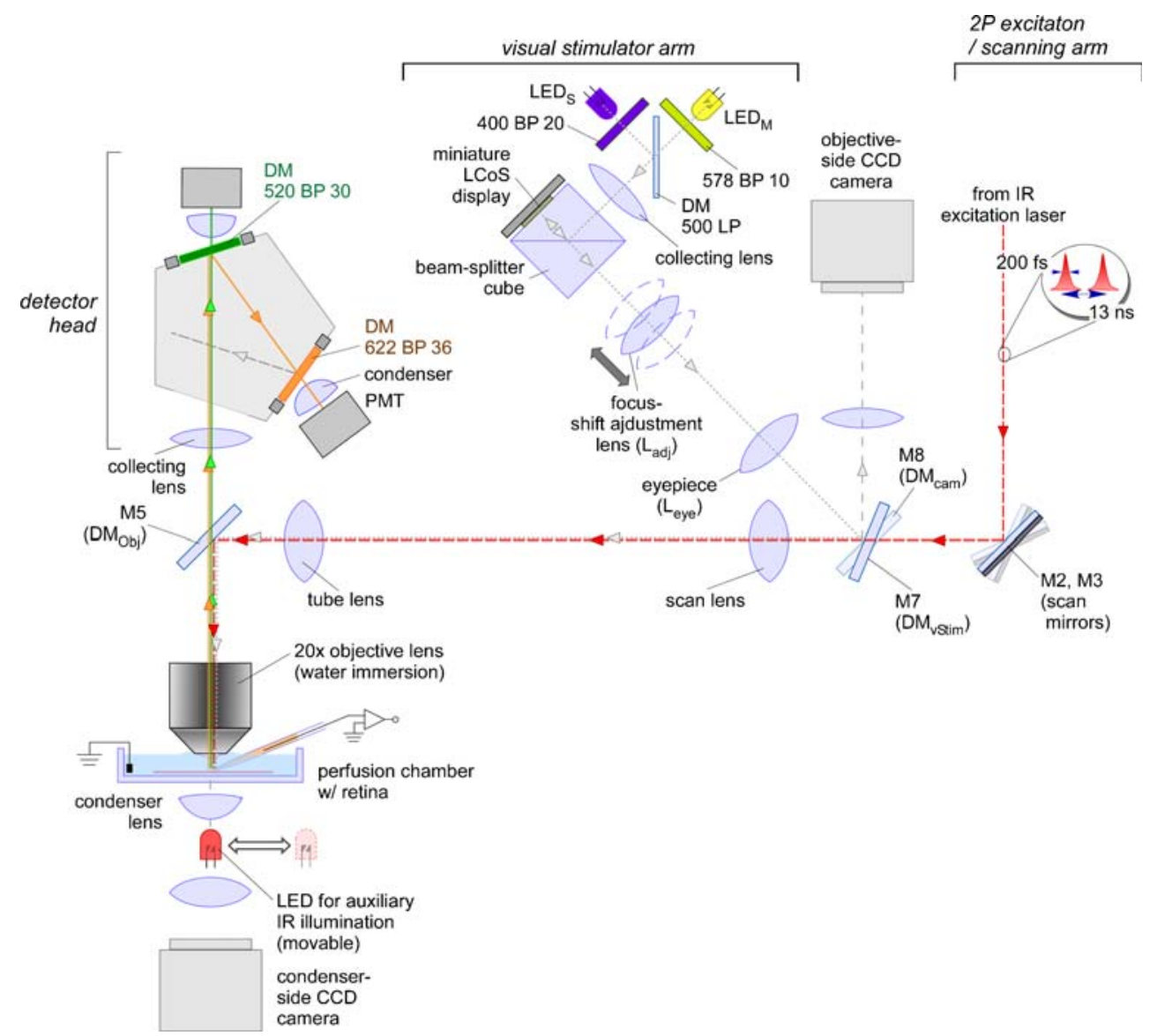


a plane equivalent to the pupil of the eye of an observer (in a conventional microscope) and enters the objective collimated. The purpose of the Eyecup scope's other (stimulator) arm is to project a focused image of an lightemitting diode (LED)-illuminated LCD onto the photoreceptor layer of the retina for visual stimulation. The light from this arm will usually enter the objective either converging or diverging, depending on whether the stimulus focus is above or below the imaging plane.

\section{Two-photon microscope}

The 2P laser scanning fluorescence microscope consists of three major parts: light source, scan system, and detector head. The following discussion of these three components also contains a description of the control electronics and software, the mechanical design, and the optical alignment of the microscope.

\section{Light source}

The light for $2 \mathrm{P}$ excitation is provided by a Ti/sapphire laser (Mira 900-F, Coherent GmbH, Dieburg, Germany) that is pumped with continuous-wave $532 \mathrm{~nm}$ light (Verdi $\mathrm{V} 10$, Coherent $\mathrm{GmbH})$. The laser generates, at $\sim 76 \mathrm{MHz}$ $\left(f_{\mathrm{p}}\right)$, ultrashort $\left(t_{\mathrm{p}}<200 \mathrm{fs}\right)$ mode-locked pulses of quasimonochromatic ( $\sim 10 \mathrm{~nm}$ bandwidth) light with a tuning range from 680 to $1060 \mathrm{~nm}$ (tuning over the full range may require changing the mirror set used in the laser cavity). Imaging with a scanning fluorescence microscope depends on being able to construct a raster "bit map" based on point-by-point measurements of fluorescence intensity. In order to do this, it is necessary that the essential properties of the pulses of light used to excite fluorescence are uniform and deliver the same excitation energy from one point to the next. This requirement is satisfied by the inherent uniformity of mode-locked laser pulses. For theory and practical operation of pulsed lasers, see [12]; for performance evaluation, see Supplemental Material 1.

There are a number of ways to regulate the intensity of the laser beam. Since the output of the Ti/sapphire laser is polarized, intensity controllers are often based on modulating the polarization state. In our instrument, this is done with a Pockels cell (Model 350-80 with model 302 driver, Conoptics), which is an electro-optic device that is placed in the beam path. It uses an applied electric field to control the birefringence of a crystal (e.g., potassium dideuterium phosphate, $\mathrm{KD} * \mathrm{P}$ ), which, in turn, modulates the polarization state of the laser beam passing through it. When combined with a polarizer at the exit port, this provides a way to make abrupt changes in laser intensity at a speed that is limited by how fast the electrical field across the crystal can be changed, this is, in particular, fast enough to attenuate the beam during the line and frame retrace periods as a way to reduce laser exposure of the tissue.

\section{Scan system}

The pulsed beam that emerges from the Ti/sapphire laser after passing the intensity control system is usually further collimated using two concave mirrors (Layertec) spaced at close to half the sum of their radii of curvature. The mirrors are arranged in a so-called z-configuration (the beam, seen from above, follows a $z$-shaped path) to keep the angles between the incoming and reflected beams on each mirror as small as possible. Slight variations of the distance between the concave mirrors change the divergence of the emerging beam. This allows the control of the laser spot diameter on the scan mirrors, which is needed to adjust the effective numerical aperture (NA) of laser illumination.

After leaving the collimator, the beam is directed (by mirror M1, see Fig. 3) to an orthogonally oriented pair of scan mirrors (M2, M3). The scan mirrors have coatings that are highly reflective in the infrared (protected silver will work but dielectric coatings optimized for the IR offer higher reflectivity and better durability and are now available from Cambridge Technology). The mirrors are fixed to independent galvanometers (Cambridge Technologies $6215 \mathrm{H}$ scanner motors), which control the XY position of the beam in the focal plane.

Laser light from the scanners enters a $50-\mathrm{mm}$ focallength scan lens (Leica Scan Okular VISIR 1534SPR136; to allow access for the stimulator beam the rear part of the lens housing was chamfered) followed by a $200-\mathrm{mm}$ focal-length tube lens (Nikon part MXA220-18) as diagrammed in Figs. 2 and 3. The scan and tube lenses translate the angular deflection of the laser beam by the movement of the scanning mirrors into a change in the angle the collimated beam enters the objective while optically transferring the pivot point from the scanners to the rear aperture of the objective. In turn, the objective converts the change in the incident angle into a change in the position of a diffraction-limited spot in the focal plane of the objective.

The distance between the scan mirror (M3) and the first principal plane of the scan lens $\left(d_{\mathrm{ms}}\right)$ is given by

$d_{\mathrm{ms}}=\frac{\left(f_{\mathrm{SL}}\right)^{2}}{f_{\mathrm{TL}}}+f_{\mathrm{SL}}-d_{\mathrm{obj}}\left(\frac{f_{\mathrm{SL}}}{f_{\mathrm{TL}}}\right)^{2}$,

where $f_{\mathrm{SL}}$ and $f_{\mathrm{TL}}$ are the focal lengths of the scan and tube lenses, respectively, and $d_{\mathrm{obj}}$ is the distance between the tube lens and the back aperture of the microscope objective, which varies, but is $95 \mathrm{~mm}$ in the center position of the $x y z$ displacement mechanism of the microscope (see below). 
Fig. 3 Technical drawings of the Eyecup scope. a CAD drawing illustrating the mechanical arrangement of the Eyecup scope, including $2 \mathrm{P}$ excitation/scanning arm, visual stimulator arm and detector head (compare Fig. 2; for abbreviations, see Fig. 2 legend). Some parts, such as excitation laser, laser conditioning optics, CCD cameras, and recording chamber, are not included. Note that the monochromatic (singleLED) version of the TTO stimulator is shown. b Same as in a but only the optical elements are shown. a, b Optical elements: cyan lenses, dark gray broad-band mirrors, lilac dichroics, bronze multicomponent optics. Mechanical parts: red commercial, gray custom-made a

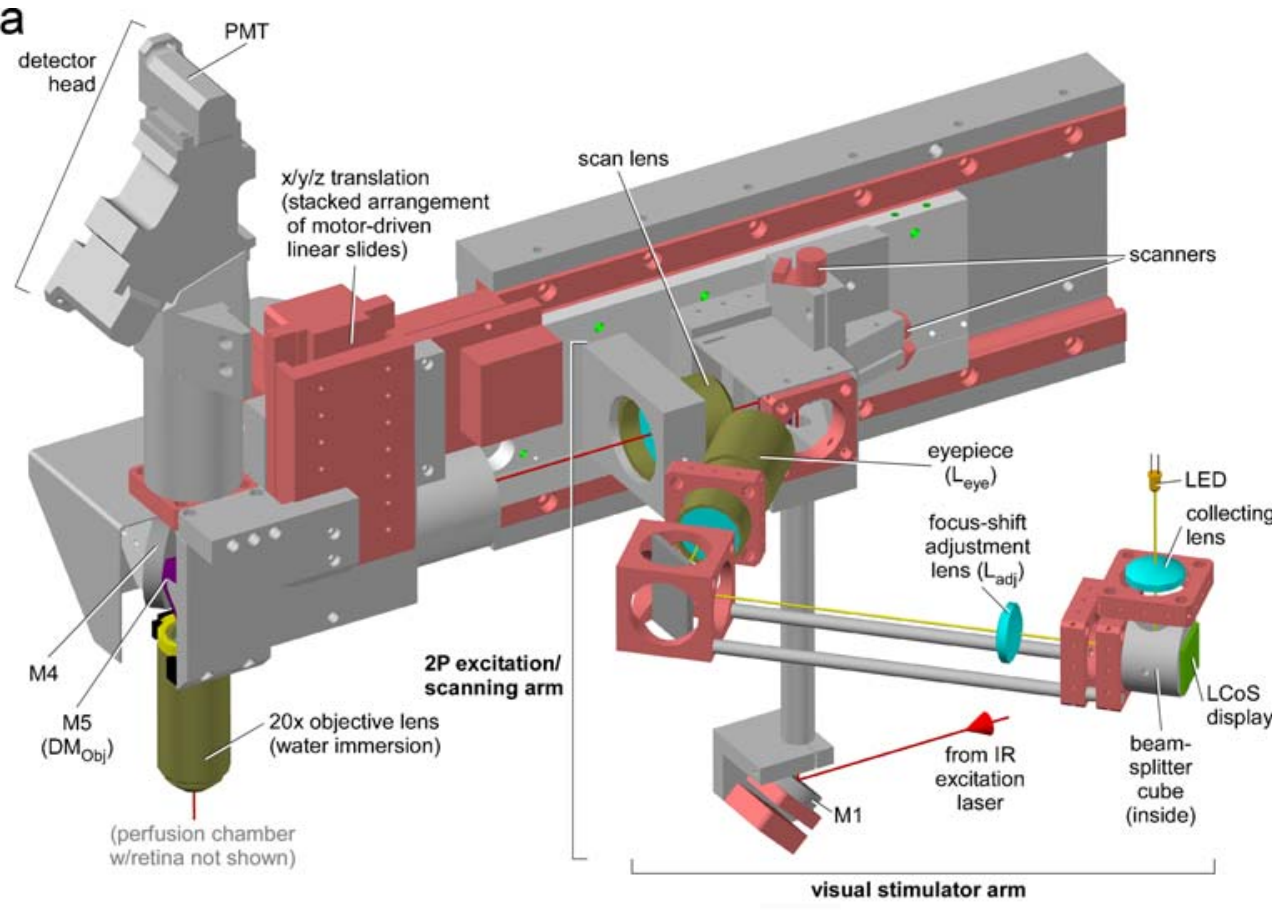

b

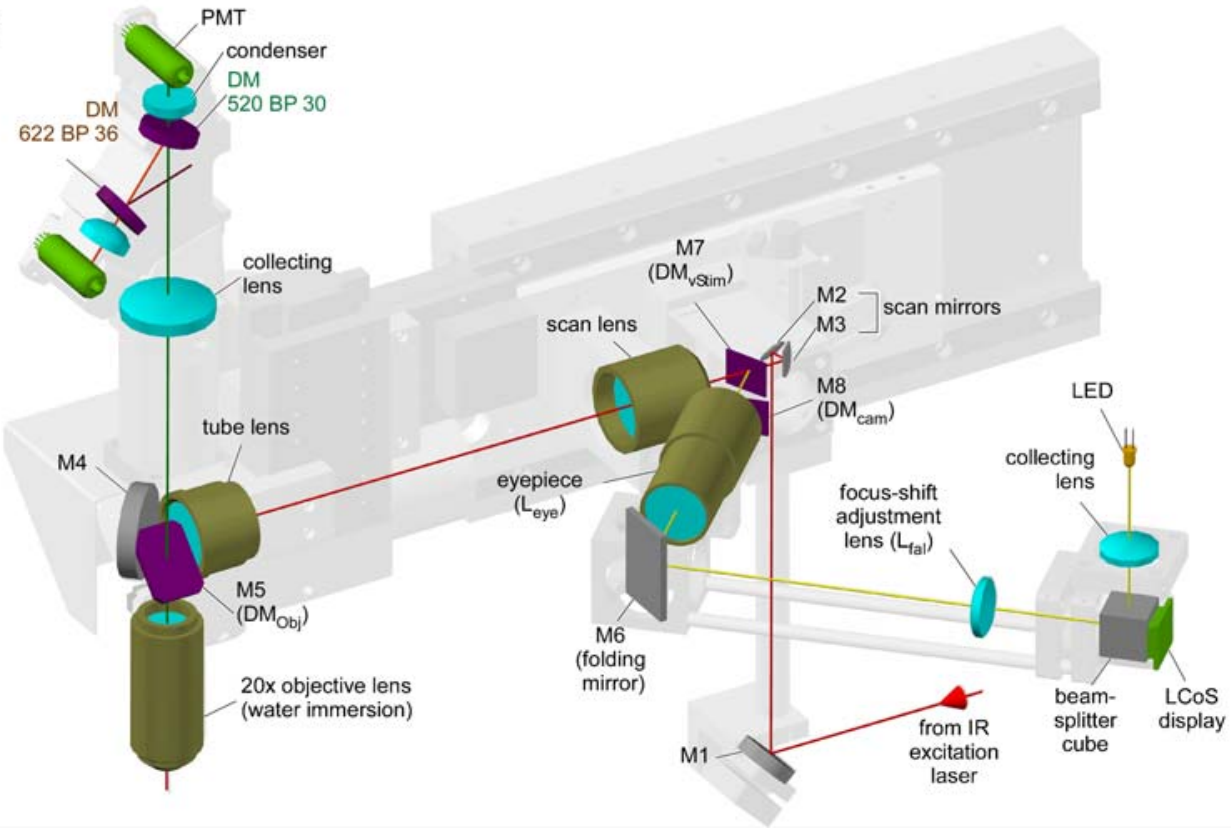

When the distance between the scan lens and the tube lens is the sum of their focal lengths $\left(f_{\mathrm{SL}}\right.$ and $f_{\mathrm{TL}}$, respectively), they act together as a telescope that magnifies the beam by

$m=\left(\frac{f_{\mathrm{TL}}}{f_{\mathrm{SL}}}\right)$.

In this configuration, collimated light entering the scan lens is focused on the common focal plane of tube and scan lens, and thus, the beam that exits the tube lens and enters the objective is collimated as well. It is also magnified (expanded) so it fills or slightly overfills the back aperture of the objective. The desirable degree of overfilling depends on a tradeoff between power throughput and resolution; for retina imaging resolution is more important than throughput and thus overfilling is favored. The required magnification depends on the ratio of the diameter of the back aperture of the objective to the diameter of the beam where it enters the scan lens. The back aperture of the objective used in the Eyecup scope (Olympus, 20× NA= 0.95 , see below) is $18 \mathrm{~mm}$ in diameter, and the magnifi- 
Fig. 4 Technical drawings of alternative mirror arrangement. a CAD drawing showing the alternative mirror arrangement (between tube lens and objective) used in a modified version of the Eyecup scope (see "Mechanical design") as well as in the in vivo scope (see text for description; for abbreviations, see Fig. 2 legend). b CAD drawings illustrating how the arrangement (a) allows independent $x y z$ translations of the objective lens (for description see text)

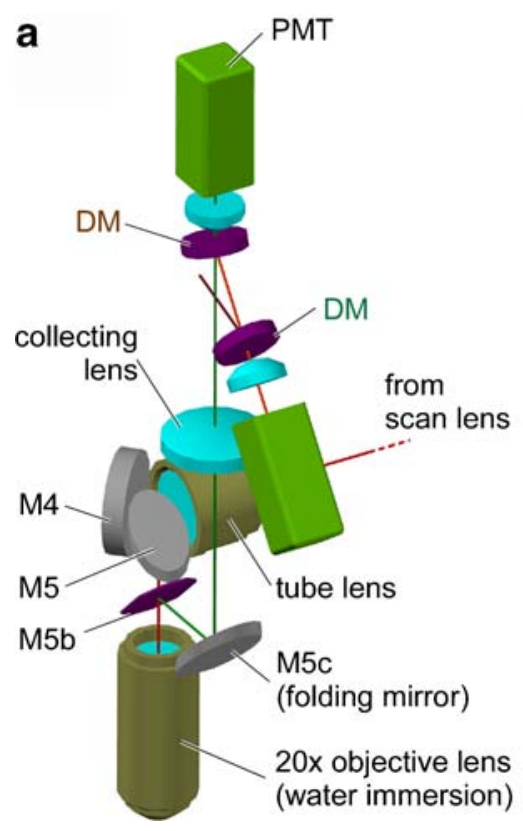

b
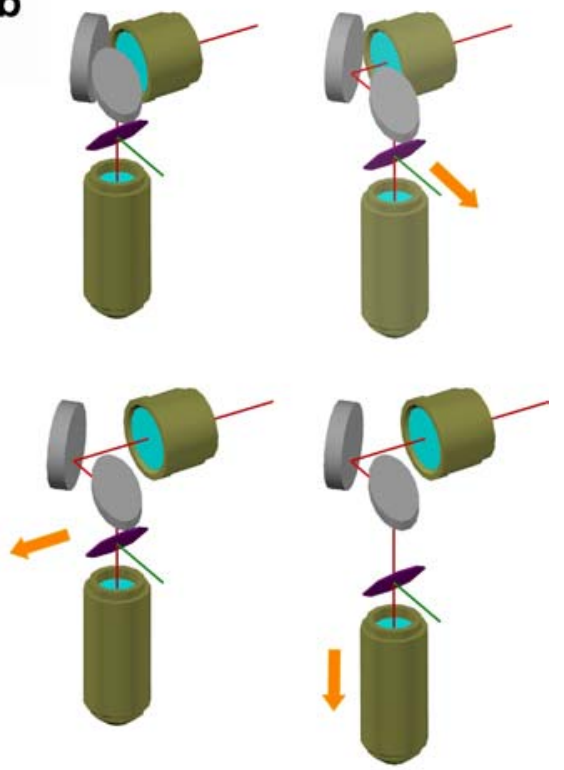

cation of the scan/tube lens telescope, which is given by Eq. 2, is $\sim 4$-fold. This means that the beam entering the scan lens needs to be at least about $4.5 \mathrm{~mm}$ in diameter to properly fill the back aperture. Therefore, 6-mm scan mirrors are used (to fill objectives with smaller back apertures, such as $40 \times / 0.8 \mathrm{NA}$ lenses, $3 \mathrm{~mm}$ mirrors are sufficient).

Light emerges from the tube lens along an axis referred to as $Y$. It is reflected $90^{\circ}$ to the $X$-axis by the first of two beam-steering mirrors. This first mirror (M4, see Figs. 3 and 4; for simplicity, not shown in Figs. 2 and 5) sends the light to the second mirror (M5) that deflects the beam $90^{\circ}$ down into the microscope objective (whose optical axis is along the $z$-axis). The objective sits below M5 and focuses the excitation laser pulses to a small spot in the retina to excite fluorophore fluorescence.

\section{Mechanical design}

The mechanical movement of the optical elements in the Eyecup scope was modified from a design developed for in vivo $2 \mathrm{P}$ microscopy (see Fig. 4a), which required that the objective position could be adjusted with respect to a stationary sample over a range of at least several millimeters in all three spatial directions without having to move the entire microscope. A solution was found involving two right-angle moving mirrors between the tube lens and the objective (Figs. $3 \mathrm{~b}$ and 4). The optical axis is turned by $90^{\circ}$ at each of the mirrors, with the optical axis of the tube lens being parallel to the plane of the retina surface (along the $Y$-axis). While the objective is translated in $x, y$, and $z$ using a stacked arrangement of (motor-driven) linear slides (MP285-3Z, Sutter Instruments), the mirror closer to the tube lens (M4) is moved along $y$ only and is fixed in $z$; the mirror closer to the objective (M5) is moved with the objective along $x$ and $y$ and is also fixed in $z$. This ensures that the optical axes of tube lens and objective stay aligned even though the distance along the optical axis between tube lens and objective changes by the sum of the displacements in $x, y$, and $z$ (Fig. $4 \mathrm{~b}$ ). Since the beam is collimated (the image at infinity) between the tube lens and the objective, a change in the distance does not degrade the imaging performance (a fact that is used in the so-called fixed-stage microscopes where the objective alone is moved when focusing). Changing the distance between tube lens and objective does, however, move the objective exit pupil away from the image of the scan-mirror pivot point (Eq. 2). This effect could become a serious concern at the outer reaches of the travel ranges $( \pm 12.5 \mathrm{~mm}$ in each direction) but can be tolerated for moderate displacements and for a limited field of view. It would be possible to eliminate the pivot-point mismatch by moving the scan mirror assembly in synchrony with the $x y z$ movements by an amount that can be calculated using Eq. 2 .

Since the detector assembly moves with the objective, detection efficiency is not affected by $x y z$ movements. Because the Eyecup scope uses through-the-objective (TTO) stimulation (see "Visual stimulator"), M5 (DM Obj $\left._{1}\right)$ is a special dichroic mirror (see "Spectral properties of stimulation LEDs, dichroic mirrors, and filters" and Fig. 5a for the spectral reflectivity) that was designed to reflect both the IR light for $2 \mathrm{P}$ excitation and the light for visual stimulation while transmitting the light emitted by fluorescence excitation. One of the difficulties is that the spectral properties of M5 $\left(\mathrm{DM}_{\mathrm{Obj}}\right)$ are angle-dependent. Because different angles in the rear of the objective correspond to 


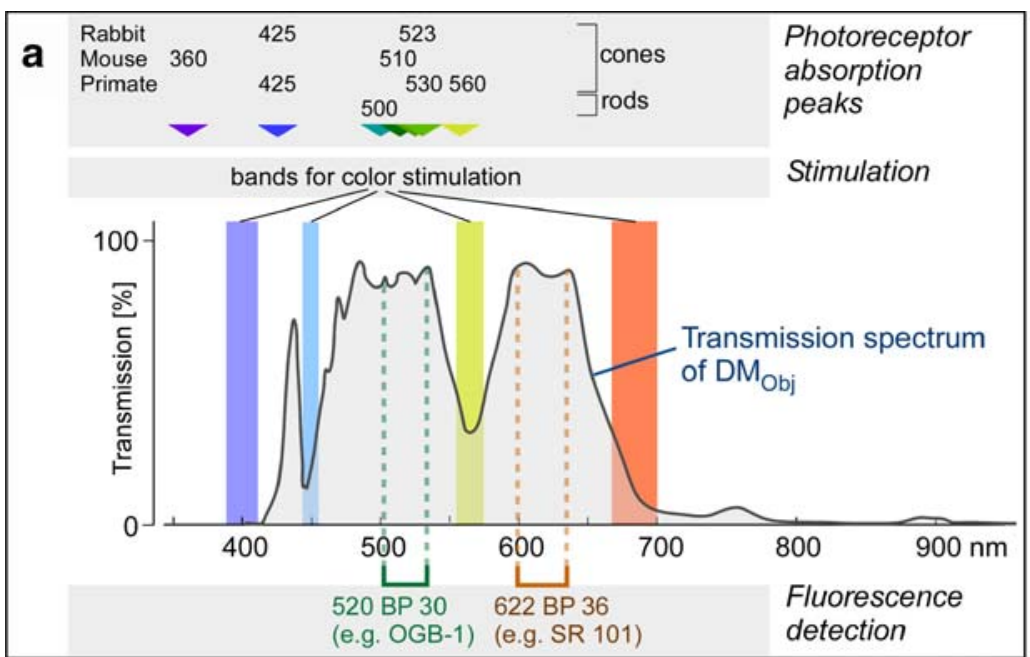

b
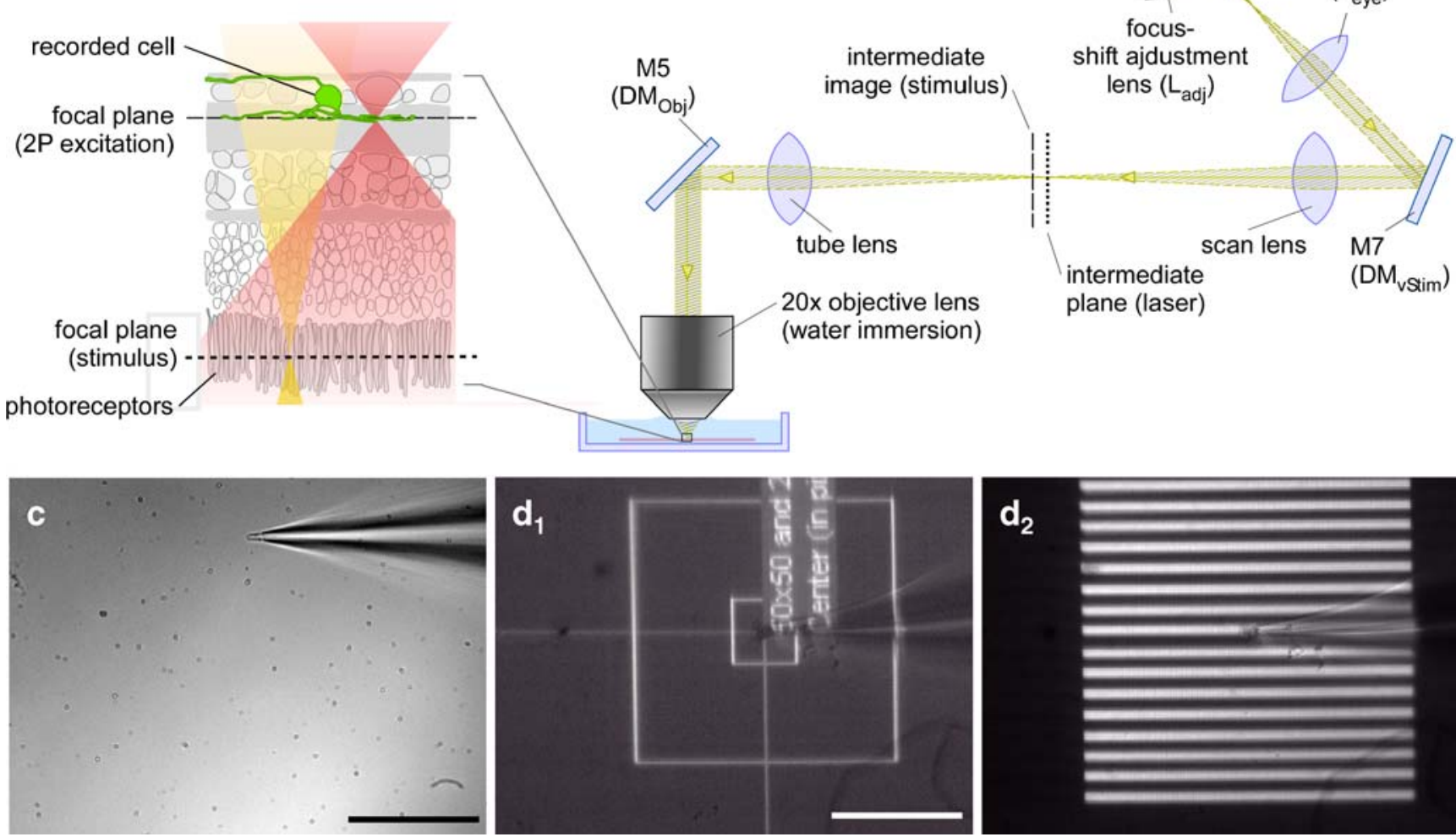

Fig. 5 Visual through-the-objective (TTO) stimulator and stimulation light path. a Spectrum illustrating arrangement of fluorescent filters and dichroic mirror $\left(\mathrm{DM}_{\mathrm{Obj}}\right)$ bands. Approximate photoreceptor absorption peaks in nm from [20,21]. b Optical path of the stimulator light. The inset to the left of the objective lens shows the different focal planes within a schematic retinal cross-section (for abbreviations, see Fig. 2). c Picture taken with the objective-side CCD camera (see Fig. 2) through a $20 \times$ lens with IR illumination from below the

different positions in the focal plane, this can result in a gradient in fluorescence collection efficiency and stimulation strength across the field of view. With the $20 \times 0.95$ NA-objective we use (see "Objective"), deviations in angle can approach $\pm 5^{\circ}$ (from the $45^{\circ}$ angle of incidence $\mathrm{DM}_{\mathrm{Obj}}$ was designed for). This results in wavelength shifts of chamber. A broken patch pipette touching the bottom of the recording chamber is visible. d Pictures taken through an oil condenser with the condenser-side CCD camera (Fig. 2) showing a patch electrode (same as in c) and different light patterns projected through the objective and focused onto the bottom of the recording chamber: a stimulus calibration pattern $\left(\mathbf{d}_{\mathbf{1}}\right.$; see "Stimulus alignment") and a stripe pattern stimulus ( $\mathbf{d}_{2}$; bright stripes, $8 \mu \mathrm{m}=4$ pixels wide) are shown. Scale bars: c, d $100 \mu \mathrm{m}$

approximately $+14 \mathrm{~nm}\left(\right.$ at $40^{\circ}$ ) and $-7 \mathrm{~nm}\left(\right.$ at $50^{\circ}$ ) for $\lambda=$ $572 \mathrm{~nm}$ (approximately in the center of the 'yellow dip' in the transmission spectrum of $\mathrm{DM}_{\mathrm{Obj}}$, see Fig. 5a). Therefore, to reduce the inevitable gradient in stimulation strength to a minimum, the LED light should be delivered in a narrow band centered on the 'dip' in $\mathrm{DM}_{\mathrm{Obj}}$. 
When detection efficiency is paramount, a slightly different arrangement (see Fig. 4) originally designed for in vivo cortical imaging can be used. It requires an additional, IR light-passing but visible light-reflecting dichroic mirror (M5b), which is inserted right above the objective (below M5) and directs the fluorescence into the detectors. This arrangement is more detection efficient because reflectivity can be made higher in dichroics than transmissivity. For the stimulus light to reach the tissue, the spectral reflectivity of M5b must contain narrow pass bands around the stimulation wavelengths (in some sense, an inverted version of $\mathrm{DM}_{\mathrm{obj}}$ ). M5 can then be an IR dielectric high reflector or a broadband metal mirror (instead of a dichroic like $\mathrm{DM}_{\mathrm{obj}}$ ). If metal mirrors are selected for both M4 and M5, this arrangement can be also used for widefield fluorescence imaging [13].

\section{Fluorescence excitation by $2 P$ absorption}

The energy of a photon is inversely related to its wavelength $\left(E_{\text {photon }}=h c / \lambda\right.$ where $h$ is the Planck constant). Thus, the energy needed to produce the electronic transition and elevate a fluorophore to the excited state can, in principle, be delivered by either a single photon of short wavelength or several, e.g., two photons for 2P absorption, at twice the wavelength [14]. For this, it is, however, necessary that the two photons are absorbed at nearly the same time (within $\sim 10^{-15} \mathrm{~s}, 1 \mathrm{fs}$, of each other). The number of photons absorbed $\left(n_{\mathrm{a}}\right)$ by one fluorophore molecule during a brief pulse lasting $t_{\mathrm{p}}$ is given by

$n_{\mathrm{a}}=\frac{p_{\mathrm{o}}^{2} \sigma}{t_{\mathrm{p}} f_{\mathrm{p}}^{2}}\left(\frac{\pi A_{\mathrm{NA}}^{2} / \lambda^{2}}{2 h c /(2 \lambda)}\right)^{2}=\frac{p_{\mathrm{o}}^{2} \sigma}{t_{\mathrm{p}} f_{\mathrm{p}}^{2}}\left(\frac{\pi A_{\mathrm{NA}}^{2}}{h c \lambda}\right)^{2}$

As Eq. 3 shows, the absorption probability depends quadratically on the number of photons that are delivered per laser pulse, which is given by the laser power $p_{\mathrm{o}}$ divided by the repetition rate $\left(f_{\mathrm{p}}\right)$ and divided by the energy of a single photon. In addition, it depends inversely on the temporal pulse width $\left(t_{\mathrm{p}}\right)$ and on how tightly the light is focused in space, which is given by $A_{\mathrm{NA}}^{2} / \lambda^{2}$, with $A_{\mathrm{NA}}$ the numerical aperture of the objective. Therefore, the probability of $2 \mathrm{P}$ excitation is increased by concentrating the delivery of photons in time, by using as short a laser pulse as possible, and in space, by using a high numerical aperture objective. In addition, the absorption probability is a function of the $2 \mathrm{P}$ cross-section of the fluorophore $(\sigma)$, which varies from one fluorophore type to the next, with $50 \times 10^{-58} \mathrm{~m}^{4} \mathrm{~s}$ per photon being a typical value [15].

Since the probability of $2 \mathrm{P}$ absorption is proportional to light intensity squared (Eq. 3), the excitation rate decays with the fourth power of the spatial distance above or below the focal point. This has the effect of carving out a small, restricted volume (less than $1 \mathrm{fl}$ ) of activation. The quadratic intensity dependence of $2 \mathrm{P}$ excitation is thus the basis of its optical sectioning ability and explains why it can be used for submicron-resolution fluorescence imaging [16].

In conclusion, $2 \mathrm{P}$ microscopy, unlike confocal microscopy, can be used for fluorescence imaging without blinding the retina: First, the wavelength of the excitation light is in the infrared and thus inefficiently absorbed by photoreceptors. Second, $2 \mathrm{P}$ absorption by the photopigment is much reduced due to the substantial distance between the focus and the light-sensitive photoreceptor outer segments, which disperses the beam over a larger area and thus reduces photon density. Third, the fluorescence, which is at wavelengths that are efficiently absorbed by photoreceptors (see "Laser-evoked effects"), is much weaker than the excitation light and is generated only in a small local volume around the focal point.

\section{Multi-channel detector head}

Fluorescence produced in the $2 \mathrm{P}$ excitation volume is incoherent and emitted in all directions. Both ballistic and scattered fluorescence photons are collected by the microscope objective and pass through the dichroic mirror M5 $\left(\mathrm{DM}_{\mathrm{obj}}\right)$ above the objective (see Figs. 2 and 3) toward the detector head as a roughly collimated beam. Since the retina is exceptionally clear, most fluorescence leaves the tissue ballistically, unlike, for example, in cerebral cortex where light is strongly scattered by neuropil. To ensure that the detector measures all the fluorescence light that exits the back aperture of the objective a collecting lens $(f=140 \mathrm{~mm}$; diameter $40 \mathrm{~mm}, 137 \mathrm{~mm}$ from the back aperture) is used to capture and focus the light into the detector head that is shown (Figs 2 and 3) with two sequentially arranged filter/ condenser/PMT combinations. The desired spectral band of fluorescence light reaches a detector by passing through a multilayer dielectric band-pass filter. These filters are oriented such that the light outside the filter's pass-band is reflected toward the next filter/detector combination. Some of the light is removed from the beam at each filter, and thus, this arrangement of filter/detector pairs is referred to as a decimation detector. It has the advantage of not requiring dichroic beam splitters in addition to the interference filters. An aspheric collector lens with anti-reflecting coating (Linos, number 317704322 ) is placed between the filter and the detector to ensure that the collected fluorescence is effectively focused onto the light-sensitive area of the detector. The distance along the optical axis between the collecting lens (below the detector head) and the center of the first filter is $88 \mathrm{~mm}$, from there to the next filter $35.5 \mathrm{~mm}$. For an objective with a back focal plane diameter of $18 \mathrm{~mm}$ and a field-of-view diameter of $1 \mathrm{~mm}$, the beam of fluorescence 
light is contained inside diameters of 18.7 and $12.8 \mathrm{~mm}$ on the first and second filters, respectively.

The detectors in the Eyecup scope are either $13 \mathrm{~mm}$, sideon photomultiplier tubes (Hamamatsu, R6357) or head-on GaAsP detectors (a special version, without cooling, of the Hamamatsu H7422P-40, now called H10770B). The latter display higher quantum efficiency, particularly between 500 and $650 \mathrm{~nm}$.

For most of the calcium-imaging experiments in the retina, the filter in front of the first detector (e.g., $510 \mathrm{BP}$ 50, Chroma HQ510/50m-2p-18deg) is optimized to detect fluorescence from a "green" (fluorescein-derived) calcium indicator, such as Oregon-Green 488 or Calcium Green-1 (Invitrogen). The filter in front of the second detector (e.g., 622 BP 36, Chroma HQ 622/36m-2p-18deg) is designed to pass fluorescence from red-emitting dyes, such as Sulforhodamine 101 (Sigma), which are used as an extracellular counterstain for cell identification and electrode targeting (see "Counterstaining living retina"). Both of these filters are manufactured by Chroma Technology and are specially designed to work at $18^{\circ}$ incidence. In the current configuration, fluorescence outside the spectral pass-band of the second filter is reflected to a 'dump', but variants of the decimation detector have been designed to allow this light to be directed to a third and fourth filter/detector combination if the experiment requires the detection of fluorescence from a third or fourth fluorophore.

\section{Electronic control and software}

The (analog) signals from the two detectors are fed to custom-designed data conditioning and digitization electronics and from there as digital signals to a frame grabber board (XPG-1000 Power Grabber with Digital Camera Module, Coreco/Dipix) plugged into a standard PC running Microsoft Windows XPTM. This PC also runs a program (CfNT, written by Ray Stepnoski, Bell Labs, and Michael Müller, MPImF) that coordinates data acquisition and generation of the voltage signals controlling the positions of the scan mirrors. Another board (Fulcrum DT 3801-G, Data Translation) in the same PC generates the voltage signals and the necessary synchronization signals, with an on-board digital signal processor ensuring the precise timing. The CfNT software also takes care of data storage, on-line display, and fast on-line analysis.

The basic operating principle of any laser-scanning microscope is to move the focused laser across the preparation in a line-by-line manner and collect the emitted fluorescence signal. The signal is filtered and integrated (either before or after digitization) and so is effectively averaged over a time interval (dwell time) that corresponds to the time the laser focus takes to traverse a pixel. While acquiring the signal with anything but the time resolution given by the detector response entails some loss of spatial resolution, this loss becomes quickly insignificant as the pixel width becomes smaller than the size of the laser focus. In addition, practical considerations (such as storage capacity or data-bus bandwidth) limit the usable data rate as well. The image is then reconstructed from the timeordered series of recorded intensity values using the knowledge of where the beam was at what time. In a raster scan, there is always a 'fast' direction (conventionally displayed as $X$-coordinate, that is in horizontal direction from left to right) and a slow direction ( $Y$-coordinate, displayed vertically from top to bottom). Usually, the speed of image acquisition is limited by the speed with which the fast-direction scanner can move mechanically, which is typically in the low kilohertz regime. High-speed recordings thus require the reduction of the number of lines (even down to a single line; line scan mode). Magnification changes (zooming) are easily implemented by scaling the scan voltages. The image can be rotated by delivering suitably scaled voltage combinations to the scanners; this allows alignment of the scan direction with elongated structures such as dendrites. Furthermore, the scan position can be changed by adding constant offset voltages. This is crucial because TTO stimulation (see "Visual stimulator") requires the objective lens to remain stationary during stimulus presentation.

The electronics were designed and custom-built to fit into a standard nuclear instrumentation module (NIM) rack and contain all the necessary circuitry for scanner controlvoltage generation, detector readout/amplification, and signal conversion. The latest version of this signal conditioning electronics [17] uses constant, high-rate (10 MHz) digitization followed by digital integration. This provides less dark-level drift and between-pixels charge loss than an analog design used in earlier versions. In addition to the two channels for fluorescence data, up to eight additional signals from arbitrary sources can be digitized once per scan line; these data are inserted into the first few pixels of each scan line. These auxiliary channels can be used to record electrophysiological data or synchronization signals (e.g., to the visual stimulus), which, by being embedded into the image file, is linked unambiguously with the fluorescence data.

\section{Alignment}

The relative positions of the optical components that make up the scanning arm of the Eyecup scope are shown in Figs. 2 and 3. The scan and tube lenses are on the same axis, which also passes though the center of the scan mirror (M3) that is closer (proximal) to the scan lens. The other scan mirror (M2) is arranged so that the reflected optical axis passes through its center if the proximal scanner is in 
its center position. The angle is chosen so that the distance between the scan mirrors' axes of rotation can be minimized while still allowing movement of the mirrors through their full angular ranges without contact between the mirrors.

The position of the scan lens can be adjusted along its axis to ensure that tube and scan lenses are separated by the sum of their focal lengths. Experimentally, this can be tested by observing whether the laser beam leaving the tube lens is collimated. The distance between the scanner assembly and the scan lens can be adjusted as well to ensure the proper placement of the pivot point in the back-focal plane of the objective (in fact, the scanner mount motion uses a lowfriction linear slide-Schneeberger, type ND2-80 - to eventually allow the dynamic correction of the pivot point shift due to $x y z$ movements of the objective, see above, Eq. 1). The distance adjustment is tested and coarsely corrected by observing whether the laser spot remains stationary on a card placed onto the back of the objective. This will not yield the exact adjustment, since the back-focal plane is usually located inside the objective. If the location is known, the card can be placed at the appropriate location. Alternatively, fine adjustment can be performed by optimizing the uniformity across a low-zoom field of view of the fluorescence from an uniform sample.

Aside from this distance adjustment, alignment of the microscope is ensured by precision machining of all parts, with a special auxiliary alignment jig that holds M4 and M5 in place while they are glued into their holders. All beam adjustments are done either while the laser is tuned to a wavelength that can be seen by the naked eye $(800 \mathrm{~nm}$ or less; care has to be taken since even quite intense light of that wavelength still looks rather dim) or by working at longer wavelengths and using either an IR viewer (e.g. Find-R-Scope 84499A, FJW Optical Systems, Palatine, IL, USA), night vision goggles (e.g., IB 1022 A, Wallfass Nachf. GmbH, Mönchengladbach, Germany), or an IR viewing card (e.g., IR sensor card, New Focus, San Jose, CA, USA). The initial alignment needed before the microscope optics can be fine tuned is to direct the laser beam onto the center of the distal (further from the scan lens) scan mirror. This is achieved using a number of adjustable mirrors (not shown except for M1 in Fig. 3) in the beam path leading from the laser to the microscope. The final steering mirror (M1), which sends the beam right to the distal scanner is actually connected to the scanner assembly and moves with it (Fig. 3). This ensures that only minor, if any, adjustments have to be made to the mirrors while the scanner position is adjusted. For this to be true, the beam has to enter M1 on an axis that is parallel to the movement direction, which is also parallel to the axis of scan and tube lens. Fine adjustments in beam position alignment can be made while observing the $2 \mathrm{P}$ fluorescence image of a uniform sample, such as dilute $(15-30 \mu \mathrm{M})$ fluorescein solution, and optimizing that image with respect to brightness and uniformity. The fluorescence signal can be shown to be generated by $2 \mathrm{P}$ excitation by interrupting mode-locking (ML) of the Ti/sapphire laser. The quadratic dependence of solution fluorescence on laser intensity can also be verified using neutral density filters to make calibrated changes in beam power: a 2-fold increase in laser intensity should produce a 4-fold increase in fluorescence intensity.

\section{Visual stimulator}

The visual stimulator arm of the Eyecup scope (Fig. 5) is designed to project an image from an illuminated screen TTO onto the photoreceptors of a retina that is placed under the objective lens.

One problem with the TTO arrangement is that the imaging plane (the objective's focal plane) will usually be located at a processing layer, e.g., the inner plexiform layer (IPL, Fig. 1a) and thus between 80 and $150 \mu \mathrm{m}$ (depending on species and retinal eccentricity) above the layer containing the light-sensitive photoreceptor outer segments (OS). While this shift between desired stimulus focus and microscope focus may appear to be small, it is many times the focal depth of a high NA objective (around one micron) and can thus by no means be ignored. By careful design, it is, however, possible to establish a defined axial offset between the focus positions (see "Stimulus image-forming pathway").

An additional requirement placed on the TTO-stimulator design is that the stimulator should not be attached to the objective-lens support so as to not increase the bulk and weight of the microscope head. We thus decided to split off the stimulation from the excitation pathways between scan mirrors (M2, M3) and scan lens (see "Stimulus imageforming pathway" and Fig. 3b). This has also the benefit that the beam splitter $\left(\mathrm{M} 7 / \mathrm{DM}_{\mathrm{vStim}}\right)$ is located at a point where the image is at infinity, and thus, astigmatism that would result from the presence of a tilted plate in the beam at a location where the beam is not collimated is avoided. To bring the beam emerging from a pixel of the stimulator screen to a focus beyond the focal plane of the objective (see above), the beam entering the scan lens has to be slightly divergent.

\section{Stimulus generation}

The stimulus screen we use is a liquid crystal-on-silicon (LCoS) spatial light modulator removed from a pair of commercial head-mounted virtual reality (VR) goggles [i-Glasses SVGA Pro, i-O Display Systems, LLC; 800× 600 pixels, 24-bit RGB color depth, compatible with Super 
Video Graphics Array (SVGA) signals]. LCoS modulators [18] are reflective displays and offer two main advantages over transmissive LCDs. The first advantage is that, unlike transmissive color LCDs, they do not contain color filters. LCoS displays such as the i-Glasses generate color images by being illuminated sequentially - within the duration of a single frame - with light from red, green, and blue LEDs synchronous with the LCoS showing the red, green, or blue frame content ('sequential color'). Thus, when these LEDs are disabled, one can choose the wavelengths (or spectral bands) of the illumination light such that it, firstly, does not overlap with the fluorescence detection bands and, secondly, allows stimulus wavelengths to be chosen for selective activation of different spectral types of cone photoreceptors (Fig. 5a). For our application, we disabled the built-in LEDs and used external LEDs for illumination (see "Spectral properties of stimulation LEDs, dichroic mirrors, and filters"). The LEDs were pulsed $(\sim 0.8 \mathrm{~ms}$ duration) in synch with the subframes by an external custom-built electronic circuit (Martin Wierny, MPImF), which was synchronized to the timing of the LCoS and controls which LED pulses during which subframe. The stimulator was used in two different modes: monochromatic (with a yellow LED pulsed three times per frame) or dichromatic (with a yellow and a blue LED each pulsed once per frame).

The second advantage is that LCoS modulators are typically faster than transmissive LCDs, allowing higher refresh rates. The display we used is built to synchronize automatically at refresh frequencies between 60 and $100 \mathrm{~Hz}$. By recording the change in light intensity reflected by the LCoS (note that due to the sequential color generation three intensity peaks occur per frame) for different full-field stimuli (e.g., alternating red/green/blue frames) using a photometer, we confirmed that no frames were dropped up to a frequency of $80 \mathrm{~Hz}$; higher frequencies were not tested. The fact that (due to the sequential color generation) the display divides every frame into three subframes can be used, in principle, to display monochromatic stimuli at a frame rate of up to $300 \mathrm{~Hz}$. This allows smoother motion stimuli, with the subframes showing intermediate phases of the movement.

\section{Stimulus image-forming pathway}

The original polarization optics of the VR goggles (see "Stimulus generation") was replaced by a polarizing beamsplitter cube (Newport, number 05FC16PB.3), which was mounted directly in front of the LCoS modulator (see Fig. 5b). The LCoS is illuminated by LEDs mounted above the beam splitter. Their light reaches the LCoS modulator polarized perpendicular to the plane of incidence on the beam-splitting surface. The LCoS has a reflective surface that is covered by a layer of nematic liquid crystal, which change the polarization of the transmitted light dependent on the locally (within a pixel) applied voltage. This acts as a voltage-dependent "light valves" because only light that is polarized perpendicular to the polarization of the incident light can pass through the beam splitter into the microscope.

To allow the distance between stimulus and imaging planes to be varied, a movable lens (focus-shift adjustment lens, $L_{\mathrm{fal}}, f_{\mathrm{fal}}=50 \mathrm{~mm}$ ) was inserted into the beam exiting the beam-splitter cube. This lens projects the LCoS modulator surface near the reticule plane of the stimulus eyepiece (see next paragraph) and is placed on a translation stage so that it can be displaced along its optical axis. Its position is read out by a linear-resistor position sensor, allowing reproducible displacements of the stimulus focus, which are needed as the microscope is focused to different depths, even during an experiment in the dark. The center position of $L_{\mathrm{fal}}$ is where the stimulation LEDs are focused and is also approximately conjugate to the back-focal plane of the objective. While the stimulus focus can be shifted, in principle, above or below the imaging focus in practice, the stimulus focus will always be below, given that meaningful $2 \mathrm{P}$ imaging is not expected in or below the photoreceptor layer (always assuming the ganglion cell layer as up). We thus adjusted the optical system such that the center position of the displacement range of lens $L_{\text {fal }}$ corresponds to a downward stimulus focus shift of approximately $100 \mu \mathrm{m}$.

Next in the optical path (aside from a folding mirror, M6 in Fig. 3b, which merely addresses a mechanical constraint in the setup) is an eyepiece ( $L_{\text {eye }}$; Leica PERIPLAN 6.3X), which serves both to place the stimulus image approximately at infinity (the exact location depends on the desired focus shift and hence on the position of $L_{\text {fal }}$ ) and to ensure that the image of $\operatorname{LED}(\mathrm{s})$ and $L_{\mathrm{fal}}$ are conjugate to the scanmirror positions and hence also the back focal plane of the objective. An eyepiece is used at this point because it is optimized to project a wide-field stimulus with good definition and large angular range.

The last step is the optical combination of the stimulus and 2P-excitation pathways. This is achieved by M7, a dichroic mirror $\left(\mathrm{DM}_{\mathrm{vStim}}\right.$, see "Spectral properties of stimulation LEDs, dichroic mirrors, and filters") that reflects the stimulus light and transmits the IR light $(930 \mathrm{~nm})$.

Since the spatial resolution needed for the stimulus is only about $2-3 \mu \mathrm{m}$ (the minimal distance between primate cones, reviewed in [3]) we need an effective NA of only about 0.15 , which we achieve by adjusting the stimulus to appropriately underfill the back-aperture and which, incidentally, also lies in the range with which the retina is illuminated in vivo. The reduced stimulus NA helps to reduce optical aberrations that are bound to result from using the objective lens at a conjugation ratio different from 
what it was designed for. The entire optical arrangement was mathematically analyzed using the so-called ABCD matrix method [19]. For details on the analysis and the resulting distances between the different optical elements see Supplemental Material 2.

One constraint for the optical arrangement is the required demagnification of the LCoS chip, which displays $800 \times 600$ pixels each $12.0 \mu \mathrm{m}(X)$ by $12.3 \mu \mathrm{m}$ $(Y)$, resulting in an active area of $9.6 \mathrm{~mm} \times 7.4 \mathrm{~mm}$. For the optical arrangement in the Eyecup scope (see Supplemental Material 2), the image of the LCoS is (de)magnified by a factor of 0.122 . If the stimulus focus is shifted from the laser focus (by moving the focus-adjustment lens, $L_{\mathrm{fal}}$ ), the magnification of the LCoS image changes (from 0.122 for the parfocal case to 0.167 and 0.210 for a stimulus planes 100 and $200 \mu \mathrm{m}$ below the laser focus, respectively, which requires $L_{\text {fal }}$ to be moved by 4.50 and $6.95 \mathrm{~mm}$ away from the LCoS chip). These demagnification factors are sufficient to ensure that for the LCD chip size of $10 \times 8 \mathrm{~mm}$, an area of more than $\sim 1 \mathrm{~mm}$ in diameter can be stimulated.

Spectral properties of stimulation LEDs, dichroic mirrors, and filters

The Eyecup scope is designed to deliver visual stimuli to the retina and at the same time use $2 \mathrm{P}$ excitation to take separate fluorescence images of the retina using two different fluorophores. For this to work, the light that reaches the two PMTs in the detector head must not be contaminated by light from either the stimulator or the laser. This is accomplished by using LEDs with appropriate emission spectra, a variety of optical filters and specially designed dichroic mirrors.

One advantage of using LCoS displays (compared to a cathode ray tube (CRT) or an organic LED (OLED) display) is that the stimulus wavelength can be easily modified (see "Stimulus generation"). Our dichromatic stimulator version is equipped with two LEDs, a yellow LED (LED ${ }_{\mathrm{M}}$, peak: $590 \mathrm{~nm}$, number 187216-62, Conrad, Hirschau, Germany) and a 'blue' LED, which peaks in the near UV (LED ${ }_{S}$, peak: $400 \mathrm{~nm}$, RLS-UV400, Roithner Lasertechnik, Wien, Austria). Before it enters the polarizing beam splitter, the light from the LEDs is band passfiltered (yellow, 578 BP 10, F34-578; 'blue', 400 BP 20; both Chroma/AHF), combined using a dichroic mirror (500 LP, Chroma/AHF \#F33-712), and collimated (Fig. 5b).

The light stimulus exits the eyepiece $\left(L_{\text {eye }}\right)$ at the end of the stimulator arm (Fig. 5b) and enters the scanning arm by reflection off mirror M7 $\left(\mathrm{DM}_{\mathrm{vStim}}\right)$ that is placed in the optical path of the scanning arm at a $22.5^{\circ}$ angle. M7 $\left(\mathrm{DM}_{\mathrm{vStim}}\right)$ reflects visible light (approximately 400 to $600 \mathrm{~nm})$ and transmits near-infrared (800 to $1,050 \mathrm{~nm})$ light. For optimal performance, the backside of M7 should have an anti-reflection coating for IR light but a standard “cold mirror" (e.g. Linos Cold Light Mirror KS 93/45 ${ }^{\circ}$ ) works as well.

The next dichroic mirror in the optical path is M5 $\left(\mathrm{DM}_{\mathrm{obj}}\right)$, which is custom-made by Chroma. This mirror sits above the objective (see "Fluorescence excitation by $2 \mathrm{P}$ absorption" and Fig. 5a,b) and is designed to reflect infrared (800 to $1,050 \mathrm{~nm}$ ) and three bands of visible light (around 440, 560, and $680 \mathrm{~nm}$ ), while being transparent to wavelengths between 480 and $550 \mathrm{~nm}$ ('green' fluorescence channel) and between 590 and $640 \mathrm{~nm}$ ('red' fluorescence channel). The three reflection bands were defined to roughly match the peak sensitivities of the three types of cone photoreceptors in primate retina (reviewed in [20]). This makes it possible to present polychromatic ("color") stimuli to the retina by selecting LEDs of appropriate wavelengths (see above). In addition, high reflectance for $\lambda<420 \mathrm{~nm}$ allows stimulation with light in the near UV range, which is useful to study chromatic processing in species with the UV-sensitive cones, such as mice [21]. The transmission characteristics of M5 $\left(\mathrm{DM}_{\mathrm{obj}}\right)$ were selected on the basis of the emission spectra of two different groups of commonly used fluorophores, fluorescein-based (green), and rhodamine-based (red) dyes. In addition, filters matching the emission characteristics of these dyes are placed in front of the detectors (see "Fluorescence excitation by $2 \mathrm{P}$ absorption") and further improve the separation of visual-stimulation light and fluorescence.

\section{Objective}

The Eyecup scope is designed to use an Olympus XLUMPlanFL 20× water-immersion, 0.95 NA microscope objective, but will likely work also with lenses of similar specifications from other manufacturers. This objective was selected for two reasons: First, it has a high numerical aperture, which is required for high-resolution $2 \mathrm{P}$ imaging (in particular in the axial direction, where it is important to suppress 2P excitation of photoreceptors, see "Laser-evoked effects"). Second, it has a large field of view (diameter, $\sim 1 \mathrm{~mm}$ ) needed so visual stimuli can be presented to an area of the retina that is substantially larger than the dendritic field of most retinal neurons (e.g., up to a few hundred $\mu \mathrm{m}$ for mouse ganglion cells, [22]).

\section{Accessory optics}

Accessory optics and charge-coupled device (CCD) cameras allow the observation of the retina in the recording chamber (mounted on the stage of the Eyecup scope) and the alignment of the stimulus. One CCD camera "looks" at the retina from above, through the objective, 
while a substage condenser delivers IR light from an LED ( $870 \mathrm{~nm}$ peak, Conrad 175322-G0) to trans-illuminate the tissue. The light collected by the objective follows the optical train of the microscope to just before the scan mirrors where it is reflected by a mirror $\left(\mathrm{M} 8 / \mathrm{DM}_{\text {cam }}\right.$ in Figs. 2 and 3; IR reflecting, e.g., Calflex X, Linos Photonics) to a monochrome CCD camera (e.g., Sony Model XC-ST70CE). The camera mirror (M8/DM $\left.\mathrm{Dam}_{\text {cam }}\right)$ and the stimulus-path coupling dichroic $\left(\mathrm{M} 7 / \mathrm{DM}_{\mathrm{VStim}}\right)$ are both mounted on a vertical roller-bearing linear slide that is moved between the two positions by a standard radiocontrol (RC) servo to select one mirror or the other. The video image from the objective-side IR camera (Fig. 2) is used to evaluate the general appearance and overall health of the retina and to locate and position the recording electrode(s) in the field of view. This can be used for both the isolated retina or the eyecup preparation. In the eyecup, however, the image of the retina is degraded and appears mottled as light used for trans-illumination passes through the semi-opaque sclera and pigment epithelium layers of the eye. The isolated retina, on the other hand, is transparent and the quality of the IR video image (Fig. 6a) is good enough for targeting and patch-clamping even small cells $(\sim 7-10 \mu \mathrm{m}$ soma diameter). For more precise morphological identification of cells and for targeting and patch-clamping cells in the eyecup preparation, laser scanning is used to obtain images by $2 \mathrm{P}$ excitation of an extracellular fluorescent counterstain (see "Counterstaining living retina") [8].

A second (condenser-side) CCD camera (Fig. 2) "looks" at the recording chamber from below and is used for aligning, calibrating, and focusing the visual stimulus. The camera is mounted under the substage condenser in place of the IR LED that provides trans-retinal illumination for the objective-side camera. For stimulus alignment, the position of the substage condenser is adjusted vertically to obtain a focused image of the top surface of the bottom of the empty recording chamber on a video monitor, with the illumination coming from the visual stimulator (for details see "Stimulus alignment").

\section{Software for visual stimulation}

Since any software that can drive an SVGA-compatible monitor output can be used with the Eyecup-scope stimulator, the user can choose from a wide variety of stimulation software. Therefore, only an overview of our software QDS (current version 3.06) will be provided in this paper. For further details, go to http://www.mpimfheidelberg.mpg.de/abteilungen/biomedizinischeOptik/softwareEntwicklung/qds/index.html.

A major requirement for any visual stimulation is timing reliability: The frames of a stimulus sequence have to be delivered to the display (see "Stimulus generation") at a defined rate (e.g., $80 \mathrm{~Hz}$ ) with minimal $(\leq 1 \mathrm{~ms})$ jitter and without dropped frames. QDS, which runs under Windows XP on most standard PCs, was designed with this requirement in mind. It relies on the precision of the refresh frequency of the graphics adapter, $i$. e., the succession of stimulus frames is coupled strictly to the succession of displayed frames. We run QDS as the only program on a dedicated PC because frame drops may occur if, due to transient high processor load (e.g., heavy network traffic), the program requires longer than the refresh interval (e.g., $12.5 \mathrm{~ms}$ at $80 \mathrm{~Hz}$ ) to generate a stimulus frame.

QDS does not require any special driver software in addition to a current version (tested with 7. $\times$ to 9.2 ) of the DirectX runtime library (Microsoft). The stimulus presentation can be started via the keyboard or triggered by an external device (e.g., the MP microscope) via a digital I/O PCI card (DIO 24 with Universal Library v5.20, Measurement Computing, Middleboro, USA). This card was also used to send trigger and/or synchronization signals from QDS to other instruments, for example, to embed stimulus markers into the imaging and electrophysiology data, to be used during off-line analysis. QDS uses the primary monitor port for displaying the user interface and the secondary monitor port (an appropriate video card must be used) for the actual stimulus presentation. In our setup, the stimulus signal is duplicated by a $250-\mathrm{MHz}$ video splitter (VM-102, Rufenach, Heidelberg, Germany). The two signals are fed, respectively, to the LCoS display in the stimulator and to a monitor on which we follow the stimulus presentation.

QDS scripts describe the presentation timing, geometry, rotation angle, size, color, brightness, motion velocity, and alpha blending of stimulus objects. These scripts can be written either in a scripting language proprietary to QDS or in Python (http://www.python.org). Every time such a script is loaded into QDS, it is executed and generates a frame-byframe stimulus 'description' which is played back during the actual stimulus presentation. Stimulus 'objects' can be simple bars, spots, annuli and sectors, or bit-mapped movies. To avoid artifacts, such as noisy edges or motion blur, movies need to be either uncompressed or compressed in a lossless fashion. Such movies can be easily created using external software; we have used ImageJ (http://rsb. info.nih.gov/ij/) and IgorPro (Wavemetrics, Lake Oswego, OR, USA).

\section{Stimulus alignment}

Stimuli are displayed on the LCoS stimulator screen relative to an origin (stimulus center) that is set in QDS (see "Software for visual stimulation"). To be able to center 
Fig. 6 Images of the retina. a A living rabbit retina wholemount viewed with the objective-side CCD camera using through-the-condenser IR illumination. Tissue surface (1), with a patch electrode over a hole in the inner limiting membrane (the hole was made by scratching the electrode sideways while applying pressure) (2), and with the focus on a cleaned ganglion cell soma (3, arrowhead). b Images at different depths of wholemounted rabbit retina recorded with the $2 \mathrm{P}$ microscope. The tissue - still alive and lightsensitive-was counterstained with the fluorescent dye Sulforhodamine 101 (SR). Layers, depth, and examples for members of the main retinal cell classes are indicated ( $m$ Müller glia; for other abbreviation, see Fig. 1; arrowheads 1, ganglion cell axon bundles; 2 pair of Müller glia processes; 3 rod bipolar cell terminal; 4 strongly SR-labeled cone pedicle; 5 rod pedicle). $\mathbf{c}$ Projection of a $2 \mathrm{P}$ image stack containing a dyefilled (with the $\mathrm{Ca}^{2+}$ indicator Oregon Green 488 BAPTA-1) direction-selective ganglion cell in rabbit retina. Cell filled via a patch electrode that was withdrawn before stack acquisition (projected stack from NFL almost through IPL; color codes retinal depth, see inset). Gray SR image (projected stack from NFL to GCL). Scale bars:

a, b $10 \mu \mathrm{m}, \mathbf{c} 50 \mu \mathrm{m}$
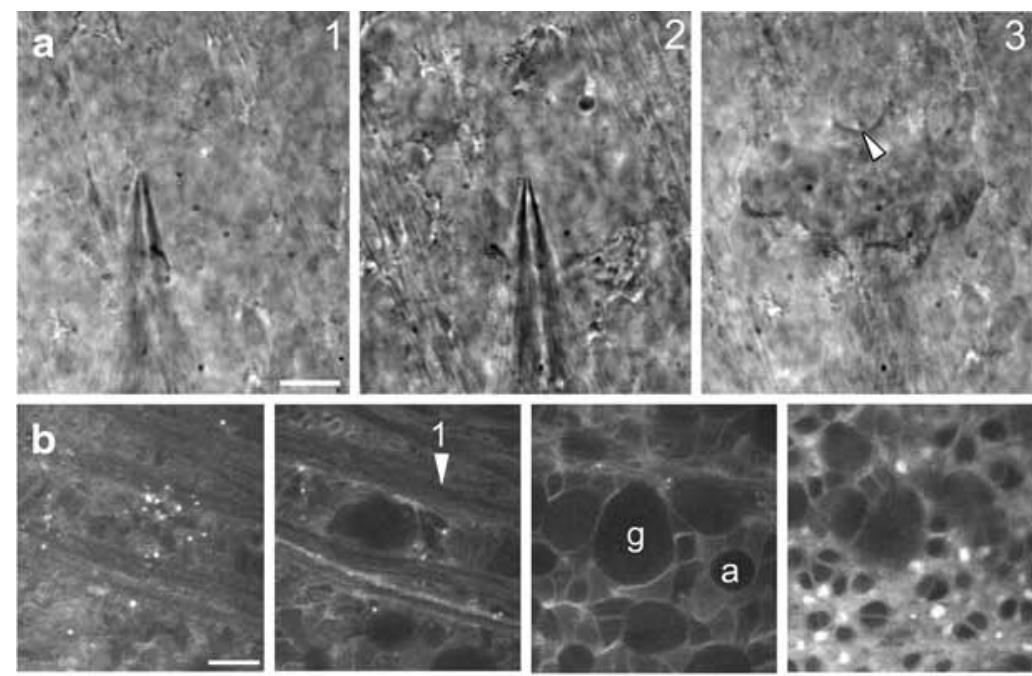

NFL

$0 \mu \mathrm{m}$

$6 \mu \mathrm{m}$ GCL

$12 \mu \mathrm{m}$

$18 \mu \mathrm{m}$
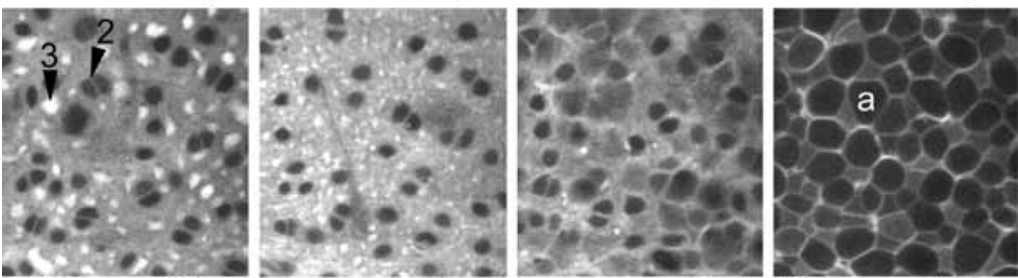

IPL

$22 \mu \mathrm{m}$

$34 \mu \mathrm{m}$

$42 \mu \mathrm{m}$ INL

$46 \mu \mathrm{m}$
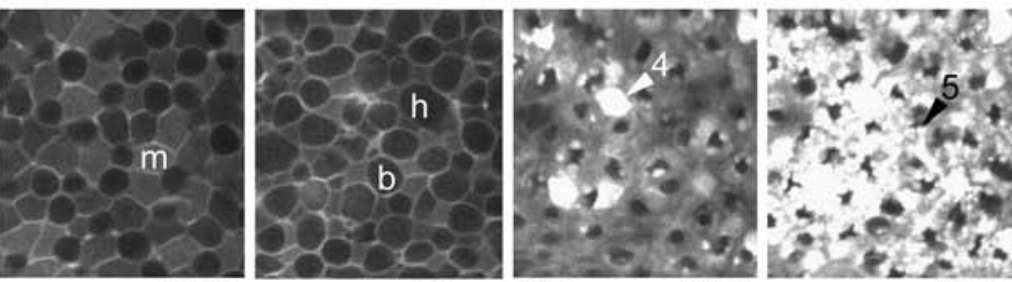

$52 \mu \mathrm{m}$

$60 \mu \mathrm{m}$ OPL

$66 \mu \mathrm{m}$

$68 \mu \mathrm{m}$
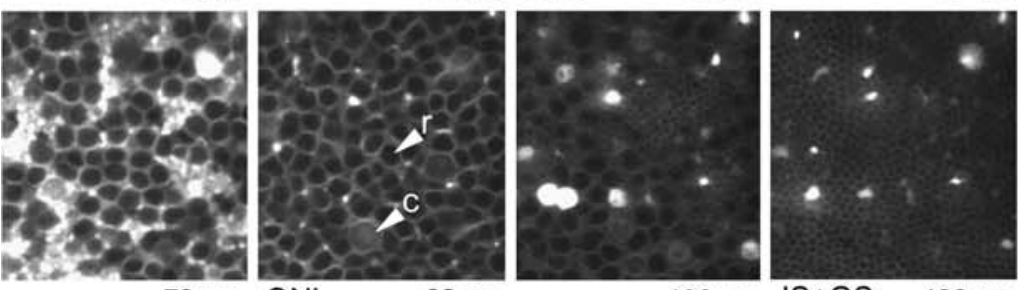

$72 \mu \mathrm{m} \quad \mathrm{ONL}$

$82 \mu \mathrm{m}$

$106 \mu \mathrm{m}$ IS + OS $\quad 120 \mu \mathrm{m}$

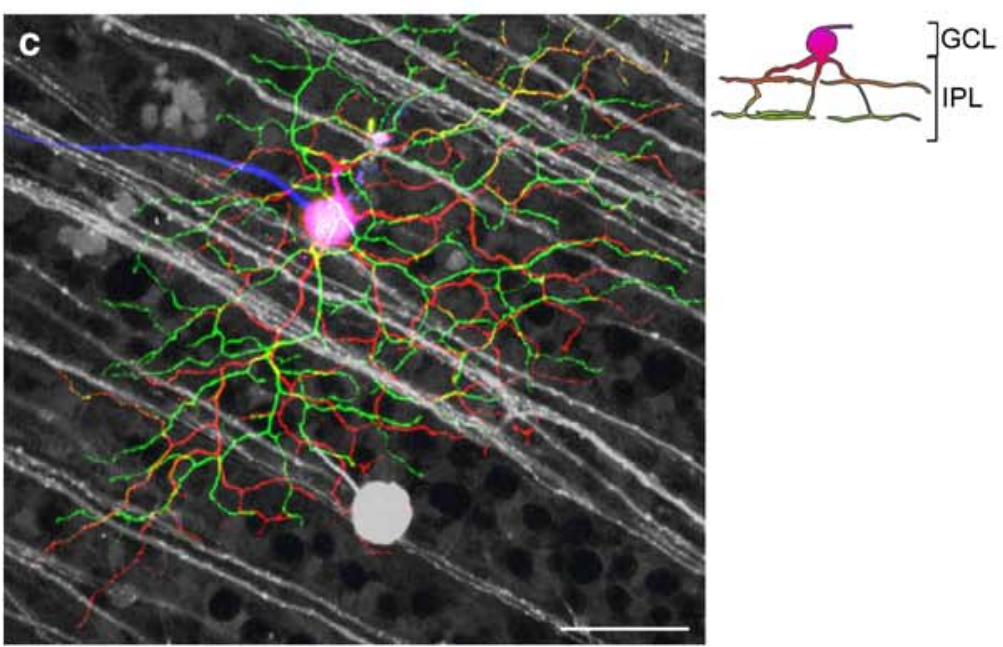


the stimulus on the recorded cell, the position of the stimulus center within the laser-scanned field of view needs to be known. Furthermore, the stimulus coordinate system has to be calibrated, such that positions and sizes are correctly scaled. The displacement between the imaging focus and the stimulus projection plane (in $\mathrm{z}$ direction) also needs to be set (by adjusting $L_{\text {fal }}$, see "Stimulus image-forming pathway"). For details on alignment and scaling procedures, see Supplemental Material 3.

\section{Stimulator performance}

In this paper, we describe the brightness and contrast of the LCoS display (see "Stimulus generation") currently used in our setup. The chip's active area of $9.6 \times 7.4 \mathrm{~mm}$ is projected into the retina such that it somewhat overfills the objective's field of view, $\approx 1 \mathrm{~mm}$ in diameter (see "Stimulus image-forming pathway").

To determine brightness and contrast of our stimulator (for the filters and LEDs given in "Spectral properties of stimulation LEDs, dichroic mirrors, and filters"), we measured the intensities (I) with the $20 \times$ objective (see "Objective") at the level of the recording chamber with an optical power meter (Model 840, Newport). Using a broadband polarizing cube (Newport, number 05FC16PB.3, 420$680 \mathrm{~nm}$, extinction ratio $>500: 1,1,000: 1$ average) and one LED pulse per frame (see "Stimulus generation"), the intensities for the yellow LED (at $578 \mathrm{~nm}$ ) ranged from $11 \times 10^{3}$ (black screen) to $255 \times 10^{3}$ (bright screen) photons $\cdot \mathrm{s}^{-1} \cdot \mu \mathrm{m}^{-2}$, and for the blue LED (at $400 \mathrm{~nm}$ ) from $41 \times 10^{3}$ to $254 \times 10^{3}$ photons $\cdot \mathrm{s}^{-1} \cdot \mu \mathrm{m}^{-2}$, corresponding to contrasts $\left(I_{\text {Max }}-I_{\text {Min }}\right) /\left(I_{\text {Max }}+I_{\text {Min }}\right)$ of $92 \%$ and $72 \%$, respectively.

Two factors contribute to the somewhat smaller contrast for the blue LED: First, the extinction ratio of the polarizing cube we used decreases for wavelengths $<420 \mathrm{~nm}$, which leads to a higher background intensity. Second, the amplitude modulation of LCDs is wavelength-dependent (e.g., [23]).

\section{Two-photon imaging of retinal neurons}

In the following sections, we describe a few of the issues relevant to using the Eyecup scope for optical recordings of visually evoked neural activity in the whole-mounted retina explant.

\section{Counterstaining living retina}

The isolated retina is transparent when viewed with IR illumination. In fact, because refractive index differences in the retina are minimal [24], presumably an adaptation to reduce scattering of light before it hits the photoreceptors, standard methods of generating images based on refractive index differences, such as Zernike phase contrast or differential interference, are much less useful in the retina than in other neural tissue. Without microdissecting the inner limiting membrane even somata in the ganglion cell layer, directly below the retinal surface can be barely recognized (Fig. 6a) and the morphological identification of cell types is usually impossible without additional labeling (e.g., 4',6-diamidino-2-phenylindole staining, see [25]), reducing the frequency of successful recordings from certain cell types.

By adding a low concentration (3 to $7 \mu \mathrm{M}$ ) of Sulforhodamine 101 (SR, Sigma) to the extracellular medium the interstitial space becomes visible in $2 \mathrm{P}$ microscopy. This provides very detailed images of the intact, light-sensitive retina (Figs. 6b,c) at an imaging rate $(4-8 \mathrm{~Hz})$ that is sufficient to guide an electrode to a selected target [8]. SR appears to be non-toxic (e.g., [26]) and can be rapidly introduced by bath application with nearly complete washout within minutes. Such counterstaining allows selected cell types to be identified on the basis of soma morphology (size, shape, and position within a retina layer) and targeted for recording with success rates as high as $90 \%$ (e.g., $[8,9,27])$.

SR labeling also allows the health of the tissue to be assessed, since it does not enter most cells in the retina unless they are damaged (Fig. 6c, gray cell soma). Some types of retinal neurons, including photoreceptors, bipolar cells, and possibly some amacrine cells, take up SR even when they are healthy; SR accumulates in synaptic terminals of photoreceptors and bipolar cells (Fig. 6b: IPL at 68 and $22 \mu \mathrm{m}$, respectively) but not in their somata (INL at $60 \mu \mathrm{m}$, ONL at $82 \mu \mathrm{m}$ ). Uptake is almost certainly active, since SR-accumulating cellular compartments are usually much brighter than SR in the medium above the tissue or in damaged cells that are filled with SR from the external solution. Activity-dependent uptake of SR into vesicles has also been reported for motor and sensory neuron endings (e.g., [28, 29]).

Counterstaining with SR does not appear to interfere with optical recordings as long as the emission from the indicator dye can be spectrally separated from that of SR, which is the case for green-fluorescing dyes, such as Oregon Green BAPTA-1. Nonetheless, to reduce phototoxicity and secondary stimulation of photoreceptors (see "Indirect excitation of retinal photoreceptors") the SR dye is normally washed out before starting physiological recordings.

Laser-evoked effects

The retina is light sensitive, and it is necessary to consider to what extent it is stimulated by the imaging 
process, either directly by the long-wavelength light used to excite a fluorophore or indirectly by the light produced by $2 \mathrm{P}$-excited fluorescence, which is emitted in all directions within the retina including in the direction of the photoreceptors. Many aspects of this issue have already been discussed by Denk and Detwiler [30]. In this paper, we describe excitation laser-evoked responses on a more practical level, with a focus on the Eyecup scope.

The onset of laser scanning typically triggers a transient response (Figs. 7b-d, 9a, but see Fig. 7e). For instance, in ON RGCs, this is a depolarization, which causes an increase in spike activity that is followed by a transient increase in $\mathrm{Ca}^{2+}$-dependent fluorescence (Fig. 9a) [transient responses often also occur at the offset of laser scanning, e.g., in OFF RGCs or even in ON RGCs cells, when the scanned area includes substantial parts of the cell's surround (Fig. 7c)]. Under optimal imaging conditions (e.g., low laser power, small field of view, sufficient background illumination; see below), the electrical activity returns within a few seconds to the pre-scanning baseline level in most of the recorded cell types. Then, electrical and $\mathrm{Ca}^{2+}$ responses can be reproducibly evoked by light stimuli (see "Light-evoked $\mathrm{Ca}^{2+}$ responses in dendrites of retinal neurons" and Fig. 9, see also [30]). If the laser power is too high, laser scanning can cause sustained activity, such as rhythmic spike activity (Figs. 7e and 8a) that is excited as the laser spot repetitively scans across (into and out of) the cell's receptive field. Because such laser-triggered activity can confound the measurement and evaluation of $\mathrm{Ca}^{2+}$ signals evoked by visual stimuli, it is crucial to understand what causes the laser effects and how to minimize them.

The different mechanisms that lead to laser-scanningevoked responses are discussed in detail in "Direct excitation of retinal photoreceptors" and "Indirect excitation of retinal photoreceptors." The most important results are summarized in the following paragraphs.

We estimate (see "Direct excitation of retinal photoreceptors") that the $1 \mathrm{P}$ excitation of the photopigments caused by a laser tuned to $930 \mathrm{~nm}$ and operating at a typical average power of $5 \mathrm{~mW}$ (in the tissue) is equivalent to an illumination of about 21 photons $\cdot \mu \mathrm{m}^{-2} \cdot \mathrm{s}^{-1}$ at $497 \mathrm{~nm}$ causing 18 activated rhodopsin $\left(\mathrm{Rh}^{*}\right)$ molecules per rod per second $\left(\mathrm{Rh}^{*} \cdot \operatorname{rod}^{-1} \cdot \mathrm{s}^{-1}\right)$. The main cause for the laser effects, however, is $2 \mathrm{P}$ excitation of the photopigments, with an equivalent illumination of about $24 \times 10^{3}$ photons. $\mu \mathrm{m}^{-2} \cdot \mathrm{s}^{-1}$ at $497 \mathrm{~nm}\left(21 \times 10^{3} \mathrm{Rh} \cdot \operatorname{rod}^{-1} \cdot \mathrm{s}^{-1}\right)$. There is also indirect photoreceptor excitation due to fluorescence emission from dye-filled cells (see "Indirect excitation of retinal photoreceptors"). Although such indirect effects can be quite substantial (e.g., Fig. 8a), we were routinely able to effectively reduce or even abolish them by scanning
Fig. 7 Laser-evoked responses in the absence of fluorescent dye. a Schematic cross-section of the retina illustrating scan size and focal planes (both for $2 \mathrm{P}$ imaging and light stimulation) relative to the recorded ganglion cell. b Spiking responses of two unidentified ganglion cells (extracellular recordings) to opening/closing of the laser shutter and to switching the laser between mode lock $(M L)$ and continuous wave $(C W)$ (scan area: $141 \times 141 \mu \mathrm{m}, 128 \times 128$ pixels/ frame, at $2 \mathrm{~ms} /$ line; stimulator background intensity $I_{\mathrm{Bkg}}=21 \times 10^{3}$ photons $\cdot \mathrm{s}^{-1} \cdot \mathrm{m}^{-2}$ ). c Spiking response of an ON ganglion cell (extracellular recording) to the laser (in ML mode) when varying the $\mathrm{z}$ position of the focal plane (as illustrated in a) (scan area and resolution as in $\mathbf{b} ; I_{\mathrm{Bkg}}=84 \times 10^{3}$ photons $\left.\cdot \mathrm{s}^{-1} \cdot \mu^{-2}\right) \mathbf{d}$ Spiking response of an ON/ OFF ganglion cell to a bright spot stimulus $(200 \mu \mathrm{m}$ in diameter) for increasing $I_{\mathrm{Bkg}}$ before $\left(\mathbf{d}_{\mathbf{1}}\right)$ and during $\left(\mathbf{d}_{\mathbf{2}}\right)$ laser scanning. Bars above traces indicate when spot was presented and when laser shutter was opened/closed. Spiking frequency as function of $I_{\mathrm{Bkg}}\left(\mathbf{d}_{\mathbf{3}}\right)$ for different time windows as indicated by the brackets below the traces (contrast $\left(I_{\text {spot }}-I_{\mathrm{bkg}}\right) /\left(I_{\mathrm{spot}}+I_{\mathrm{bkg}}\right)$ between stimulus spot and background: $32 \%, 39 \%, 36 \%, 33 \%, 27 \%, 16 \%, 13 \%, 10 \%$, and $4 \%$. Scan area: $23 \times 23 \mu \mathrm{m}, 256 \times 256$ pixels at $2 \mathrm{~ms} /$ line) e Spiking response of an ON ganglion cell; same conditions as in $\mathbf{d}$ except that the spot diameter was $800 \mu \mathrm{m}$ and the scan area (zoom) was varied. Only traces for two different $I_{\mathrm{Bkg}}$ are shown. Laser: tuned to $\sim 925 \mathrm{~nm}$; mode locked $(M L)$ except in b; power: $\mathbf{b}, \mathbf{c}, \mathbf{e} \sim 12 \mathrm{~mW} ; \mathbf{d} \sim 7 \mathrm{~mW}$; focal plane in the GCL except in c. Stimulator LED: yellow, band pass-filtered (578 BP 10) and used for both stimulus and background

small areas of retina and/or avoiding highly fluorescent structures.

Taken together, our results indicate that, largely due to 2P photopigment excitation, optical recordings in the retina are restricted to the mesopic/photopic range. Next to working with as little laser power as possible, the most effective way to minimize laser effects is to use light adaptation via steady background illumination to reduce the overall light sensitivity of the retina. It is also important to recognize that not all cell types have the same sensitivity to laser stimulation, which makes it necessary to independently assess the laser sensitivity of any cell type that is studied using $2 \mathrm{P} \mathrm{Ca}^{2+}$ imaging or electrophysiological recordings.

\section{Direct excitation of retinal photoreceptors}

Direct stimulation by the laser can arise from either 1P or 2P absorption by the receptor photopigment. Some direct stimulation by the laser via $1 \mathrm{P}$ absorption of the photopigment is inevitable. However, its likelihood falls off strongly with increasing wavelength, and therefore, $1 \mathrm{P}$ absorption can be drastically reduced by using longer excitation wavelengths beyond the peak of the photopigment absorption spectrum $[31,32]$.

The 1P excitation by the IR beam can be estimated using the limiting slope of sensitivity at decreasing photon energy (decreasing frequency, increasing wavelength) [33]. The normalized sensitivity $S$ (with intensities expressed as photon fluxes) of a photopigment can be described as a 


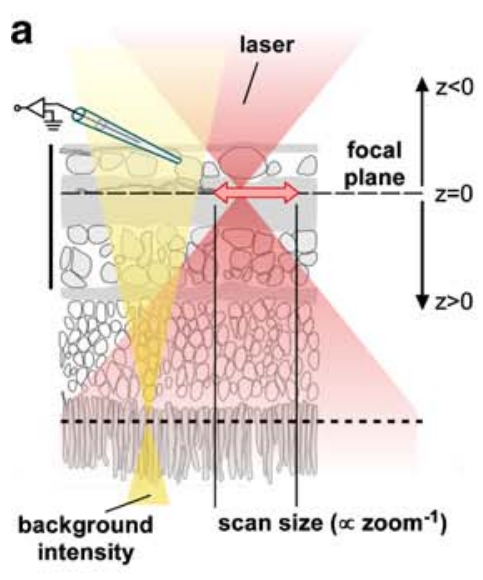

b

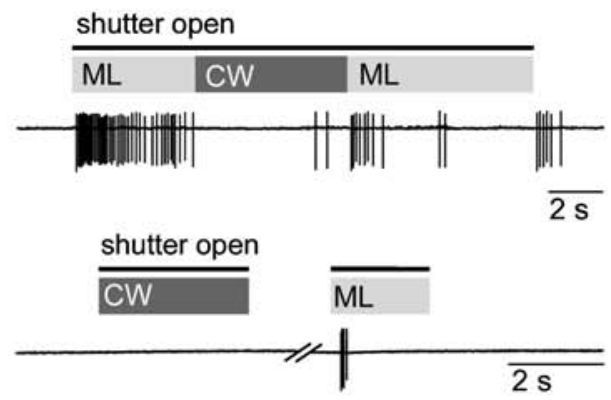

C

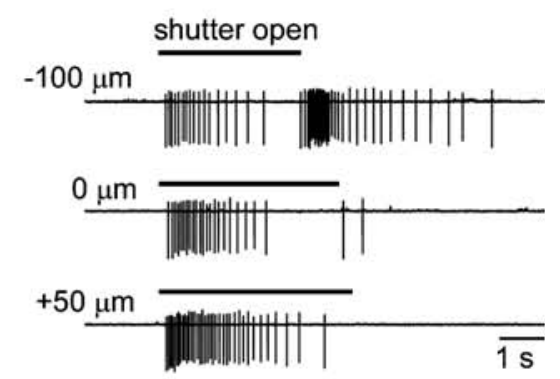

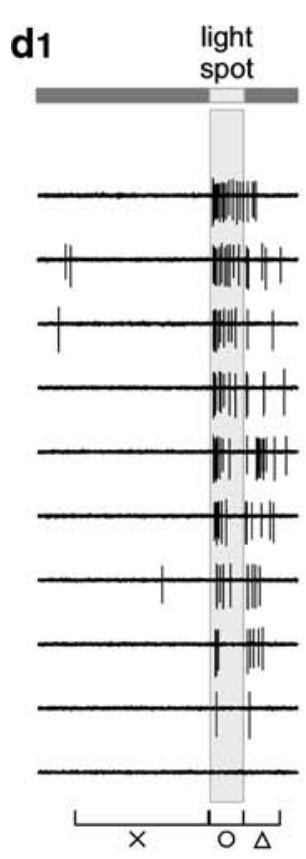

d2
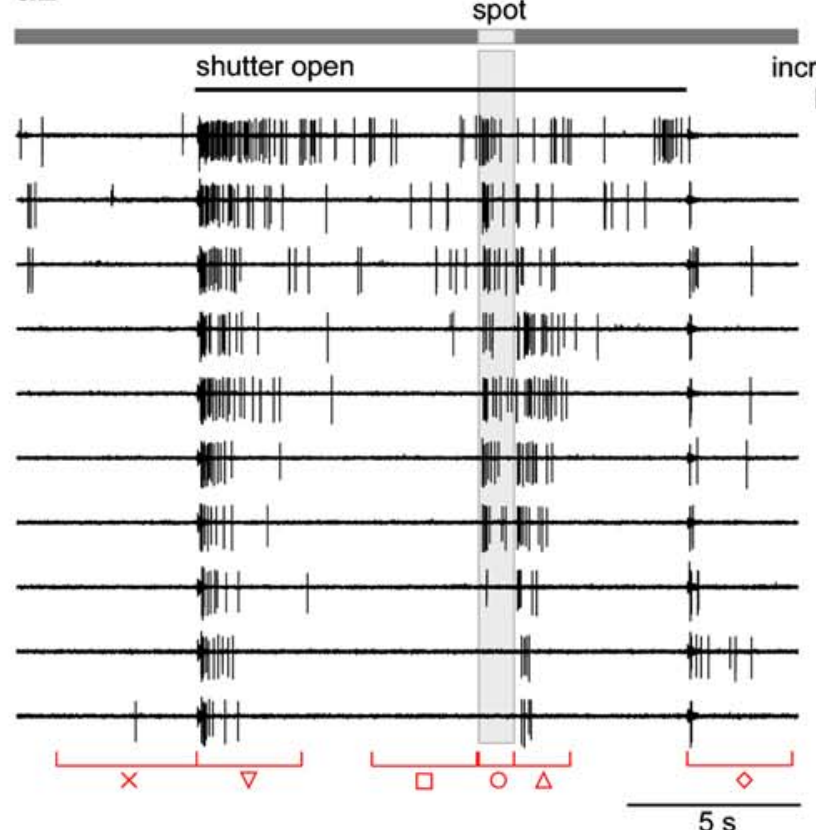

e1

light spot

shutter open

scan area $\sim 23 \times 23 \mu \mathrm{m}$

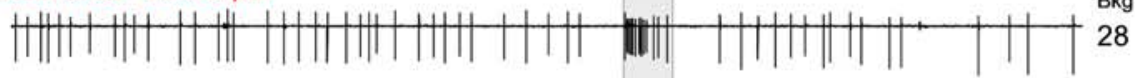

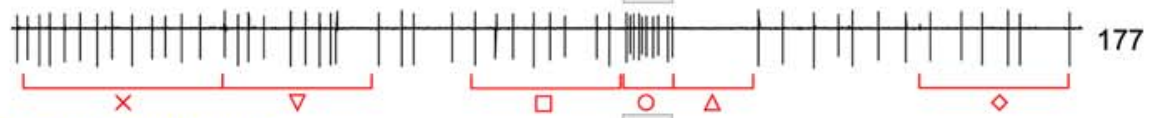

scan area $\sim 81 \times 81 \mu \mathrm{m}$

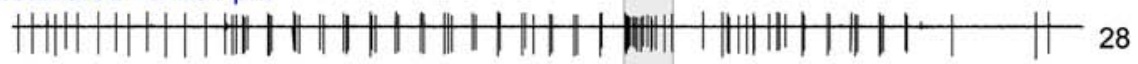

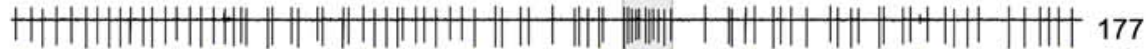

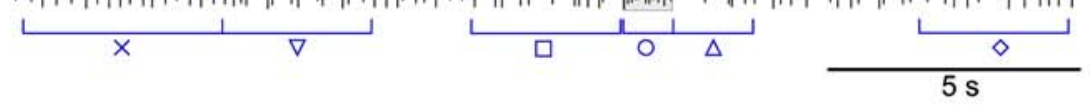

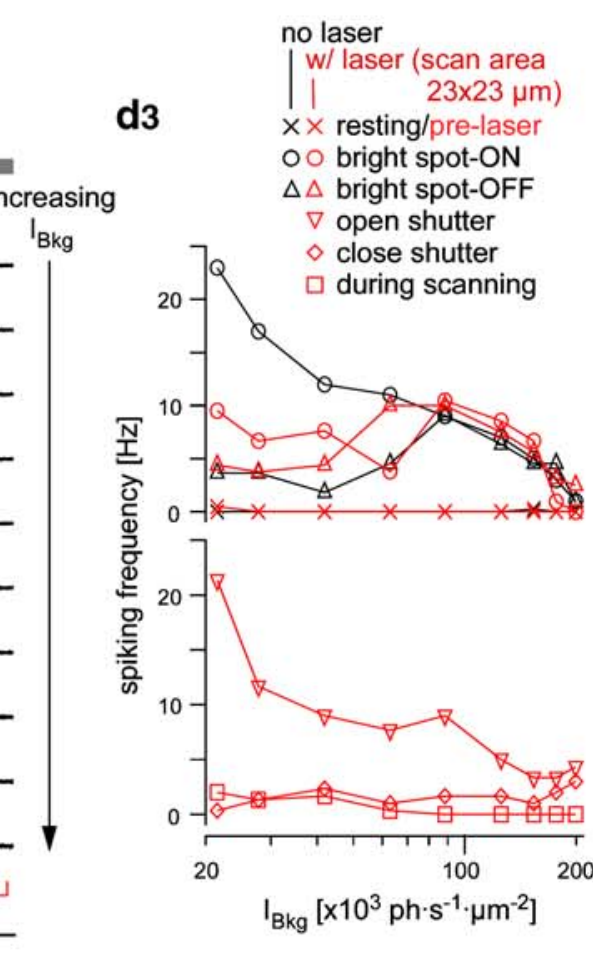

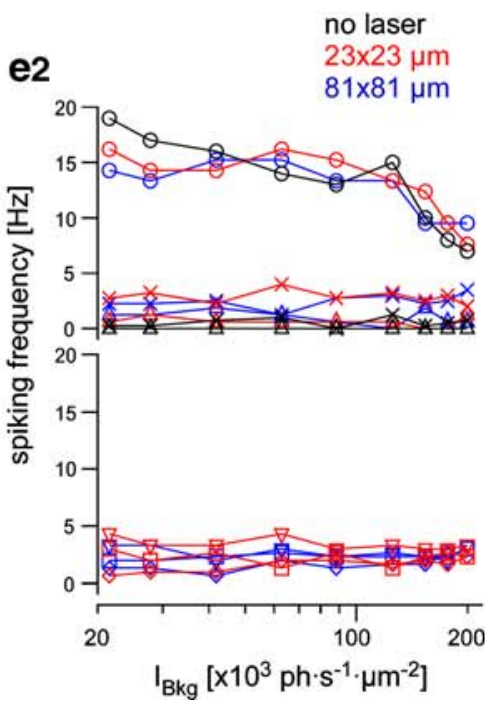


a

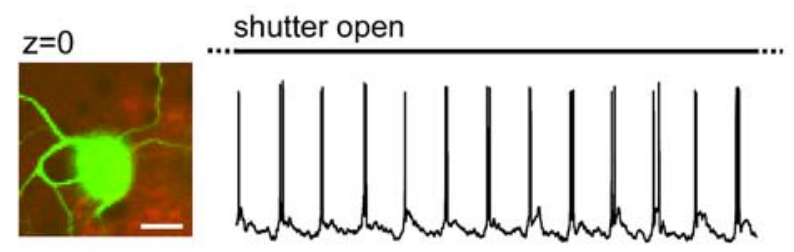

b

shutter open

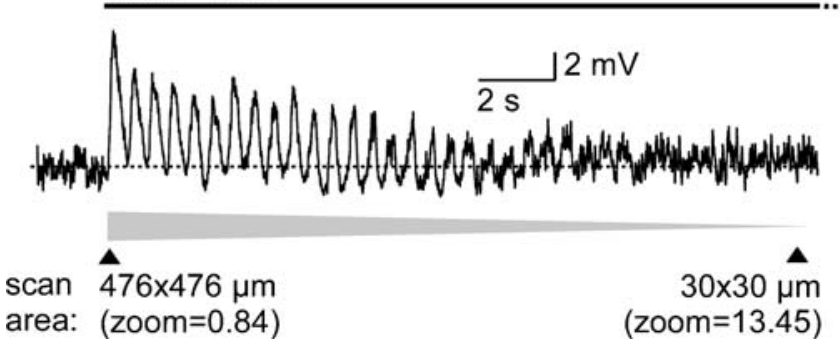

Fig. 8 Laser-evoked responses with fluorescence dye in tissue/ recorded cell. a Voltage response of a direction-selective ON/OFF ganglion cell, which was recorded and filled with OGB-1 via a patchclamp electrode. The cell spikes periodically to its own somatic fluorescence when the laser scans the soma (left trace). Shifting the focus below the soma eliminated this response (right trace). (images: green OGB-1; red extracellular Sulforhodamine 101; scale bar: $20 \mu \mathrm{m}$. Scan area: $84 \times 84 \mu \mathrm{m}, 128 \times 128$ pixels/frame, at $2 \mathrm{~ms} / \mathrm{line}$; $I_{\mathrm{Bkg}}=53 \times 10^{3}$ photons $\left.\cdot \mathrm{s}^{-1} \cdot \mu \mathrm{m}^{-2}\right)$. b Voltage response of a starburst amacrine cell (SAC) recorded with a patch-clamp electrode (cell filled with OGB-1) to the laser when continuously decreasing the scan area (bottom gray trace) by changing the zoom factor. Scan area: variable,

function of normalized frequency $x=v / v_{\max }\left(=\lambda_{\max } / \lambda\right)$ using Eq. 1 from Lamb [33]

$S(x)=\left\{A \exp \left(-\frac{a x}{n}\right)+B \exp \left(\frac{b x}{n}\right)\right\}^{-n}$,

which, using $a=72, b=8$, and $n=4$ (with $A=b /(a+b)$ $\exp (a / n)$ and $B=a /(a+b) \exp (-b / n))$, adequately fits the experimentally measured pigment absorption (for details, see [33]). This means, for example, that the rod sensitivity at $930 \mathrm{~nm}$ is smaller by a factor of $2.75 \times 10^{-11}$ (using Eq. 4) compared to the maximal sensitivity (at $497 \mathrm{~nm}$ in mouse retina). The total excitation by a $5-\mathrm{mW}$ beam $\left(2.5 \times 10^{16}\right.$ photons $\mathrm{s}^{-1}$ ) at $930 \mathrm{~nm}$ beam therefore corresponds to an illumination with about $0.14 \mathrm{pW}\left(1.38 \times 10^{-13} \mathrm{~W}=\sim 7 \times 10^{5}\right.$ photons s${ }^{-1}$ ) at $497 \mathrm{~nm}$. Because of the distance between the focal plane of the laser beam in the IPL and the photoreceptor outer segments $\left(d_{\mathrm{fp} \text {-pos }}\right)$, the illumination is distributed over an area, which can be calculated using

$\tan \left[\arcsin \left(\frac{A_{\mathrm{NA}}}{n_{\text {refr }}}\right)\right]=\frac{r}{d_{\mathrm{fp}-\mathrm{pos}}}$

(with the objective's $A_{\mathrm{NA}}=0.95$ and the refractive index $n_{\text {refr }}=1.33$ for water). For $d_{\text {fp-pos }}=100 \mu \mathrm{m}$, the illuminated

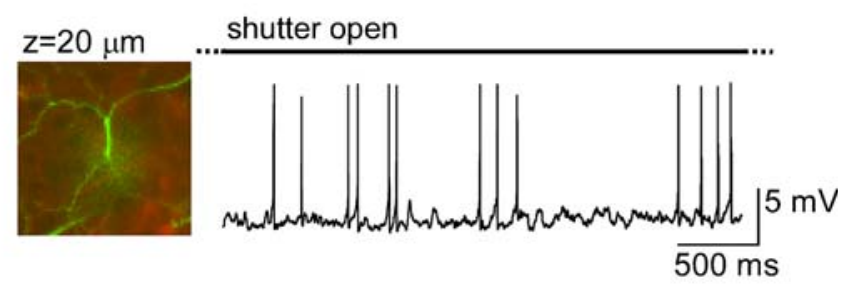

C

shutter open

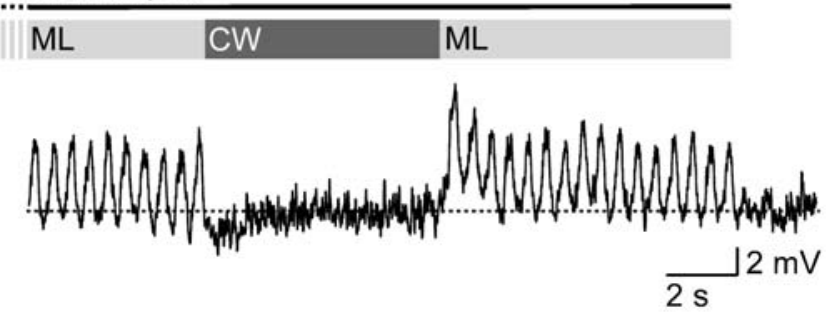

$128 \times 128 \mathrm{pixels} /$ frame, at $2 \mathrm{~ms} /$ line; $I_{\mathrm{Bkg}}=21 \times 10^{3}$ photons $\cdot \mathrm{s}^{-1} \cdot \mu \mathrm{m}^{-2}$. c Voltage response of a SAC recorded with a patch-clamp electrode (and filled with OGB-1) to switching the laser between mode lock $(M L)$ and continuous wave $(C W)$. Conditions as in $\mathbf{b}$ except scan area, which was $168 \times 168 \mu \mathrm{m}$. Fluorescent staining: intracellular solution with 100-200 $\mu \mathrm{m}$ OGB-1; extracellular Sulforhodamine 101 largely washed out of tissue except in a (there $\sim 5 \mu \mathrm{M}$ ). Laser: tuned to $\sim 925 \mathrm{~nm}$; mode locked $(M L)$ except in c; power: $\sim 12 \mathrm{~mW}$; focal plane in the IPL (except left trace in a). Stimulator LED: yellow, band-passfiltered (578 BP 10) background illumination. For more details on recording methods, see [9]

Fig. 9 Recording light stimulus-evoked activity. a Simultaneously recorded dendritic $\mathrm{Ca}^{2+}$ (red trace, from time-lapsed image scan with $64 \times 8$ pixels/frame and $2 \mathrm{~ms} /$ line) and somatic voltage (black trace) in an ON DS ganglion cell $\left(\mathbf{a}_{\mathbf{1}}\right)$. Responses to opening the laser shutter and to a bright, moving bar stimulus (for details see b) are plotted. Single scan frames $\left(\mathbf{a}_{2}\right)$ of the light stimulus-evoked response in a dendrite and magnified laser-evoked response $\left(\mathbf{a}_{\mathbf{3}}\right)$ at the beginning of the recording are also shown. $\mathbf{b}$ Schematic drawing $\left(\mathbf{b}_{\mathbf{1}}\right)$ illustrating the visual stimulus (bright moving bar, $200 \times 500 \mu \mathrm{m}, 1,000 \mu \mathrm{m} / \mathrm{s}$ ) in relation to an $\mathrm{ON} /$ OFF DS ganglion cell, which was recorded via patch electrode. Yellow arrow indicates motion direction of bar stimulus; scan position marked by red line. $\mathbf{b}_{2}$ Simultaneously recorded dendritic $\mathrm{Ca}^{2+}$ (red trace, from line scan data shown above with 64 pixels/line and $2 \mathrm{~ms} / \mathrm{line}$ ) and somatic voltage (black trace) of the cell in response to a bar moving in preferred direction (p.d.) and 'null' direction (n.d.). $I_{\mathrm{Bkg}}=42 \times 10^{3}$ photons $\cdot \mathrm{s}^{-1} \cdot \mu \mathrm{m}^{-2}, I_{\text {Stim }}=194 \times 10^{3}$ photons $\cdot \mathrm{s}^{-1} \cdot \mu \mathrm{m}^{-2}$. c Drawing $\left(\mathbf{c}_{\mathbf{1}}\right)$ illustrating the visual stimulus (a circular wave) in relation to a starburst amacrine cell, which was recorded as described in a. Scan position marked by red box. $\mathbf{c}_{2}$ Dendritic $\mathrm{Ca}^{2+}$ signal (red trace from time-lapsed image scan with $64 \times 8$ pixels/frame and $2 \mathrm{~ms} / \mathrm{line}$ ) and somatic voltage response (black trace) to the centrifugally (expanding) or centripetally (contracting) moving stimulus (from Fig. 1e in [9], modified). Single frames of image scan are shown above traces. Fluorescent staining: patch-clamp electrodes contained 100-200 $\mu \mathrm{m}$ OGB-1; extracellular Sulforhodamine 101 largely washed out of tissue. $\mathrm{Ca}^{2+}$ signals: as $\Delta F /$ $F_{0}$ after subtracting background fluorescence; from image scan $(\mathbf{a}, \mathbf{c})$ or line scan (b) data. Laser: tuned to $\sim 925 \mathrm{~nm}$; mode locked; power: 3 $6 \mathrm{~mW}$; focal plane in the IPL. Stimulator LED: yellow, band passfiltered (578 BP 10) for stimulus and background. For more details on recording methods, see [9] 


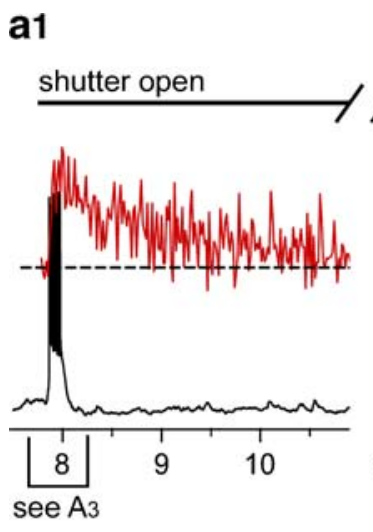

b1

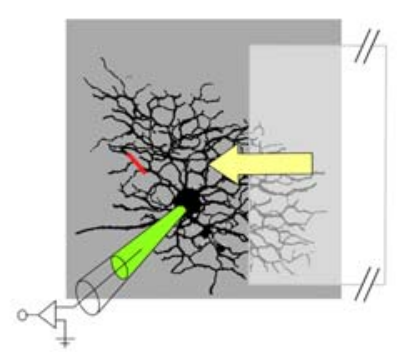

moving bar (n.d.)

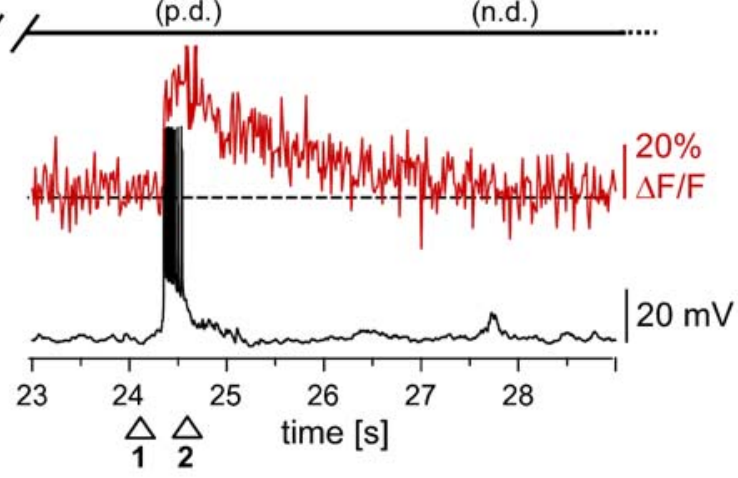

a2
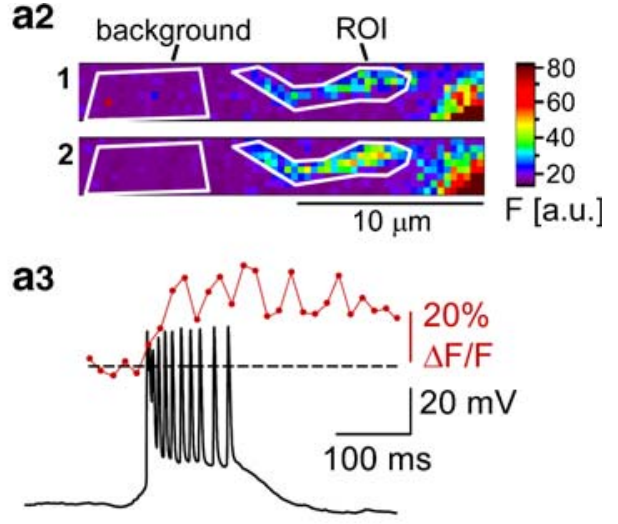

b2

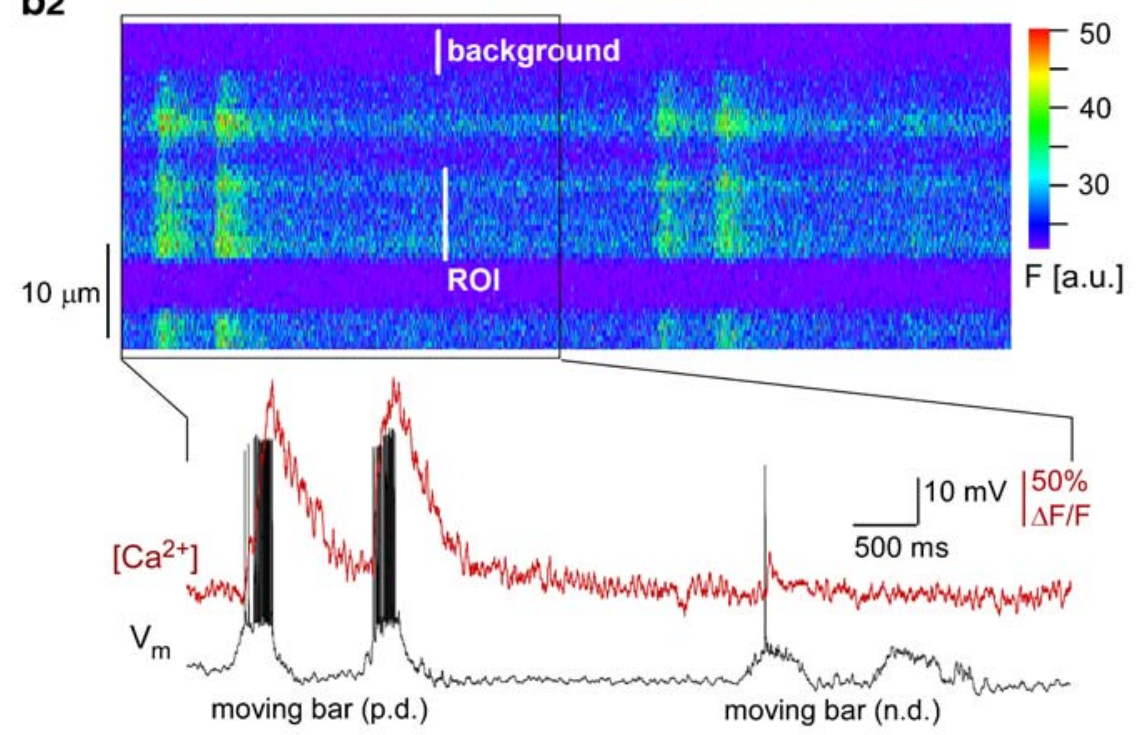

C2

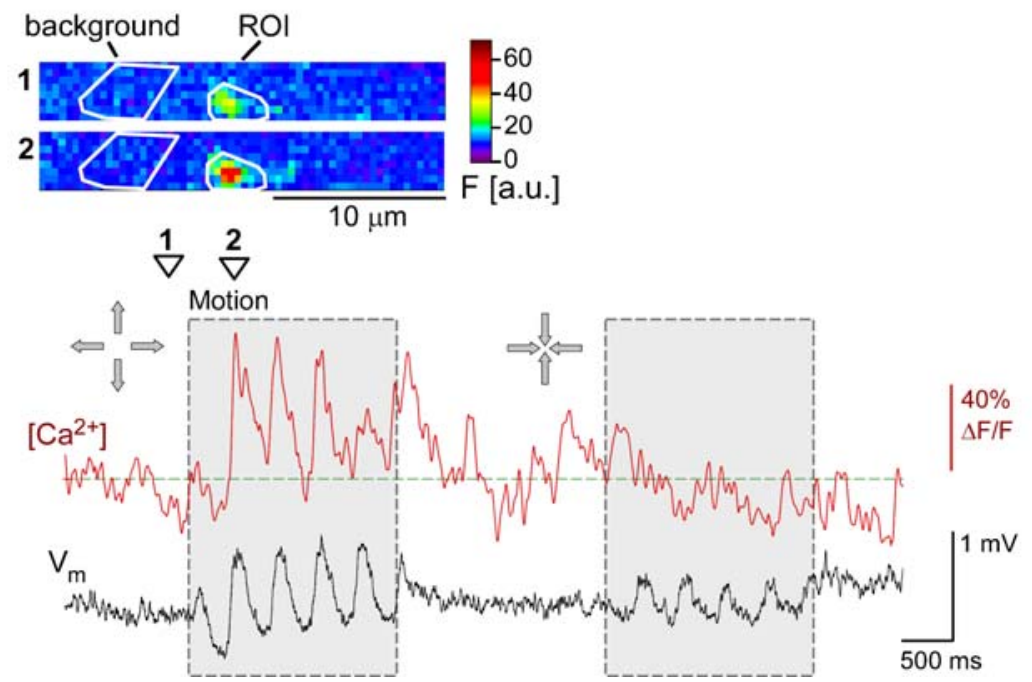

spot has a radius $(r)$ of $102 \mu \mathrm{m}$ and an area $\left(A_{\mathrm{POS}}\right)$ of $3.3 \times$ $10^{4} \mu^{2}$. Thus, the intensity at the photoreceptors is $\sim 21$ photons $\cdot \mu \mathrm{m}^{-2} \cdot \mathrm{s}^{-1}$ at $497 \mathrm{~nm}$, which corresponds to $\sim 18 \mathrm{Rh} * \operatorname{rod}^{-1} \cdot \mathrm{s}^{-1}$ using an effective collecting area of
$0.87 \mu^{2}$ for axial illumination of mouse rod [34]. While there is little dependence of the total $1 \mathrm{P}$ excitation on the focal position, as the focus moves deeper into the retina, the diameter of the area of illuminated photoreceptors decreases 
and there is a corresponding increase in photon flux density (photons $\mu \mathrm{m}^{-2}$ ).

To estimate the direct $2 \mathrm{P}$ excitation of the photopigment, we assume that visual pigments have a $2 \mathrm{P}$ cross-section, $\sigma$, comparable to that of typical fluorophores, i.e., around $50 \times$ $10^{-58} \mathrm{~m}^{4} \cdot \mathrm{s} /$ photon [15]—-some measurements estimate substantially larger numbers for rhodopsin (e.g. [35]). The rate with which each pigment molecule undergoes $2 \mathrm{P}$ absorption events is then

$$
\begin{aligned}
R & =\sigma\left\langle\frac{p_{0}}{A_{\mathrm{POS}}}\right\rangle^{2} \xi \\
& =50 \times 10^{-58}\left(\frac{\lambda}{h c}\right)^{2}\left(\frac{5 \times 10^{-3}}{3.3 \times 10^{-8}}\right)^{2} 10^{5} \approx \frac{0.0003}{\mathrm{~s}}
\end{aligned}
$$

where $\xi=\left(t_{\mathrm{p}} f_{\mathrm{p}}\right)$ (see "Light source:) is the $2 \mathrm{P}$ "advantage" [36]. Under these conditions in a mouse rod with $7 \times 10^{7}$ rhodopsin molecules [34], the rate of rhodopsin activation by $2 \mathrm{P}$ absorption for a $5-\mathrm{mW} 930-\mathrm{nm}$ beam would be $21 \times$ $10^{3} \mathrm{Rh}^{*} \cdot \operatorname{rod}^{-1} \cdot \mathrm{s}^{-1}$, corresponding to an intensity of $\sim 24 \times$ $10^{3}$ photons $\cdot \mathrm{mm}^{-2} \cdot \mathrm{s}^{-1}$ at $497 \mathrm{~nm}$. It is important to note that this is an overestimate, as it ignores e.g. light scattering and the dependence of photoreceptor sensitivity on the angle of incident light [37-39]. Taken at face value, this is more than 1,000 times the 1P excitation, rate and (among the direct effects) 2P excitation of the photopigments is therefore expected to be the main source of the laserevoked activity. In fact, $2 \mathrm{P}$ excitation in the outer segments of isolated rods has been demonstrated [40].

To evaluate the effects of direct photoreceptor excitation by the laser, the electrical activity of background lightadapted RGCs was recorded extracellularly in the absence of any fluorescent dye (Fig. 7). Experimentally, 2P excitation should be distinguishable from $1 \mathrm{P}$ excitation as being dependent on whether the laser is mode locked or not $\left(\xi \approx 10^{5}\right.$ vs. $\left.\xi=1\right)$. That $2 \mathrm{P}$ excitation is an important factor for laser-evoked activity becomes evident when switching the laser between mode lock (ML) and continuous wave (CW) mode: RGCs typically responded to the laser when in ML but not when in CW mode (Fig. 7b). Nonetheless, under completely dark adapted conditions (without stimulator background), we found that mouse RGCs that are mainly rod photoreceptor-driven, such as $\alpha$-cells [41], exhibit substantial responses to CW-laser scanning (data not shown) - consistent with the estimate of 1P photopigment excitation above.

Varying the focal plane position changes the response in a way that is consistent with the accompanying change in the photoreceptor area illuminated (Fig. 7c). As the focal plane moves inward (away from the photoreceptor layer resulting in a larger illuminated area), the laser-OFF response increases for ON RGCs, likely reflecting an increase in inhibitory input from the surround. With the focal plane closer to the photoreceptors (fewer photo- receptors illuminated), the laser-OFF component disappears and the laser-ON response increases.

The laser-evoked responses depend strongly on the level of steady background illumination provided by the light stimulator (Fig. 7d,e). In these experiments, we compared the spiking responses of RGCs to a spot stimulus with and without laser scanning. In each of these cells, the spot diameter was adjusted to elicit the maximal response, and the scan area and laser power were set at values typically used for making dendritic $\mathrm{Ca}^{2+}$ measurements. We found substantial differences in the recorded RGC's susceptibility to the laser scanning (cp. Fig. 7d,e). Presumably, this reflects differences between RGC types. In most cases, sufficient background intensity from the stimulator eliminated the sustained laser-evoked activity (including the periodic spiking that occurs for larger scan areas; e.g. Fig. 7e1, lower two traces), such that the RGCs' light stimulus-evoked responses without laser became indistinguishable from those during laser scanning. Increasing the stimulator background typically reduced but did not abolish transient laser-evoked responses. This points out the need to insert a time delay (3-5 s) between the onset of laser scanning and the first presentation of a visual stimulus.

We recorded also from RGCs that showed reduced responses during laser scanning even at the highest stimulator background intensity tested (data not shown), which was $\sim 200 \times 10^{3}$ photons $\cdot \mu \mathrm{m}^{-2} \cdot \mathrm{s}^{-1}$. The maximal intensity our current stimulator can provide $(\sim 750 \times$ $10^{3}$ photons $\cdot \mu \mathrm{m}^{-2} \cdot \mathrm{s}^{-1}$, with three pulses per frame, see "Stimulator performance") was not used as a background to limit photopigment bleaching.

\section{Indirect excitation of retinal photoreceptors}

Stimulation by fluorescence emission can be substantial when the scanned area includes a highly fluorescent structure, such as the soma of a dye-filled cell. We can estimate the effective illumination intensity caused by the excited fluorescence using the following: To generate a signal-to-noise ratio of, say, 30 for each scanned line, we need to detect at about 1,000 photons/line, which, for a total detection efficiency of $5 \%$ and a scan rate of $2 \mathrm{~ms} / \mathrm{line}$, is equal to $\sim 10^{7}$ generated photons $\cdot \mathrm{s}^{-1}$. Since these photons are emitted isotropically, they will also excite photoreceptors. When coming from dyefilled dendrites in the IPL $\sim 100 \mu \mathrm{m}$ away, the intensity at the level of the photoreceptors is at most $\sim 80$ photons. $\mu \mathrm{m}^{-2} \cdot \mathrm{s}^{-1}\left(10^{7}\right.$ photons $\cdot \mathrm{s}^{-1}$ divided by the surface of a sphere, $4 \pi\left(d_{\mathrm{fp} \text {-pos }}\right)$, with $\left.d_{\mathrm{fp} \text {-pos }}=100 \mu \mathrm{m}\right)$, which corresponds to $70 \mathrm{Rh}^{*} \cdot \operatorname{rod}^{-1} \cdot \mathrm{s}^{-1}$. This is $\sim 300$ times less than the equivalent intensity caused by direct $2 \mathrm{P}$ photoreceptor stimulation (see "Direct excitation of retinal photoreceptors").

Indirect photoreceptor stimulation by fluorescence emission can be minimized by restricting scanning to smaller 
cell compartments that emit less fluorescence (Fig. 8a). Restricting the scan area reduces time-varying photoreceptor stimulation because for small area scans or line scans (unlike for the slower large-scale area scans), the temporal periodicity in the generated fluorescence is so fast that the resulting illumination is practically constant causing the retina to be locally light adapted. This is apparent when comparing responses of a SAC to scans at different zoom factors (Fig. 8b).

In conclusion, several measures can be taken to eliminate or at least reduce indirect and direct laser-evoked effects: (1) light-adapting the retina using background illumination,( 2) restricting scans to small areas or short lines, (3) avoiding highly fluorescent structures (e.g., somata), and washing out any counterstain (such as Sulforhodamine). Under these conditions, laser-evoked responses can usually be reduced such that they no longer interfere with the light stimulus-elicited responses (see "Light-evoked $\mathrm{Ca}^{2+}$ responses in dendrites of retinal neurons," Fig. 9, and $[8,9,27])$.

Light-evoked $\mathrm{Ca}^{2+}$ responses in dendrites of retinal neurons

While the Eyecup scope is useful for taking high-resolution 3D morphological data sets of retinal neurons (see "Counterstaining living retina", Figs. 1 b,c and $5 b, c$ ) without blinding the retina, it was designed primarily to optically record light stimulus-evoked responses in neural compartments (e.g., distal dendrites) too small to be recorded from in other ways (see $[8,9,27,30,42-44]$ ).

Dendritic $\mathrm{Ca}^{2+}$ signals in response to light stimulation were recorded from ganglion cells and SACs (red traces in Fig. 9, examples from rabbit retina). Cells were filled with $\mathrm{Ca}^{2+}$ indicator via a patch electrode, which was also used to simultaneously record the cell's somatic voltage response (black traces). Retinal cells have also been successfully filled with dye (by diffusion or iontophoresis) using sharp microelectrodes, which usually were retracted after a few minutes. In both cases, some time is required for the dye to reach distal dendrites (between 15 and $60 \mathrm{~min}$, depending on the size of the dendritic arbor).

We recorded fluorescence signals from small areas using either line scans (for best temporal resolution) or small image scans. It is important to keep in mind that image acquisition is entirely sequential, i.e., one pixel at a time. For example, when scanning a line across a dendrite (Fig. 9b), the indicator fluorescence signal is low at the beginning when the laser pulses are focused on unstained cells or the extracellular space (which may be stained with SR but is free of the indicator dye), then abruptly increases as the excitation beam crosses into the dye filled dendrite only to drop back to baseline again when the scan beam leaves the dendrite on the opposite side. We typically use a scan rate of $500 \mathrm{~Hz}(2 \mathrm{~ms} /$ line $)$ with a $1.6-\mathrm{ms}$ forward sweep, during which the fluorescence data are acquired and a 0.4-ms retrace. To reduce photo damage, the Pockels cell intensity controller (see "Light source") may be used to blank the laser during the retrace portion of the line scan. For background subtraction, the scan line may be positioned to include an area free of indicator dye to provide background-reference data at essentially the same time as the fluorescence measurement (see Fig. 9b,c, background).

When temporal resolution is less important, the imagescan mode offers a way to reduce the laser exposure that each point receives (minimizing bleaching and phototoxic effects) by integrating the fluorescence signal over a larger area, e.g., a stretch of dendrite or a group of terminals (Fig. 9c). A typical image scan consists of frames with eight lines (64 pixels each) at $2 \mathrm{~ms} /$ line (resulting in $62.5 \mathrm{~Hz}$ frame scan rate). By using different regions of interest (ROIs), the fluorescence signals in different parts of the imaged branch can be compared.

In conclusion, these results show that a single objective lens can be used to deliver visual stimuli and record multi photon-evoked fluorescence signals from the dendrites of retinal neurons, structures too small to record from electrically.

Acknowledgments We thank M. Hauswirth, M. Müller, J. Tritthard, and M. Wierny for technical assistance, J. Dübel, A. Gartland, and P. Theer for testing, and J. Binding and W. Mittmann for comments on the manuscript. This study was supported by the Max-Planck Society, by the German Research Foundation (DFG) Research Unit 701 (TB, TE), by NIH grant EY002048 (PBD), and Human Frontiers Science Project Grant RGP0067 (SH, PBD).

Open Access This article is distributed under the terms of the Creative Commons Attribution Noncommercial License which permits any noncommercial use, distribution, and reproduction in any medium, provided the original author(s) and source are credited.

\section{References}

1. Kerr JN, Denk W (2008) Imaging in vivo: watching the brain in action. Nat Rev Neurosci 9:195-205

2. Masland RH (1986) The functional architecture of the retina. Sci Am 255:102-111

3. Wässle H, Boycott BB (1991) Functional architecture of the mammalian retina. Phys Rev 71:447-480

4. Masland RH (2001) The fundamental plan of the retina. Nat Neurosci 4:877-86

5. Wässle H (2004) Parallel processing in the mammalian retina. Nat Rev Neurosci 5:747-57

6. London M, Hausser M (2005) Dendritic computation. Annu Rev Neurosci 28:503-532

7. Euler T, Denk W (2001) Dendritic processing. Curr Opin Neurobiol 11:415-422 
8. Euler T, Detwiler PB, Denk W (2002) Directionally selective calcium signals in dendrites of starburst amacrine cells. Nature 418:845-852

9. Hausselt SE, Euler T, Detwiler PB, Denk W (2007) A dendriteautonomous mechanism for direction selectivity in retinal starburst amacrine cells. PLoS Biol 5:e185

10. Masland RH (2001) Neuronal diversity in the retina. Curr Opin Neurobiol 11:431-436

11. Denk W, Strickler JH, Webb WW (1990) Two-photon laser scanning fluorescence microscopy. Science 248:73-76

12. Gosnell TR, Taylor AJ (1991) Selected papers on ultrafast laser technology. Milestone series. SPIE, Bellingham

13. Wachowiak M, Denk W, Friedrich RW (2004) Functional organization of sensory input to the olfactory bulb glomerulus analyzed by two-photon calcium imaging. Proc Natl Acad Sci USA 101:9097-9102

14. Göppert-Mayer M (1931) Über Elementarakte mit zwei Quantensprüngen. Ann Phys (Paris) 9:273-295

15. Xu C, Webb WW (1996) Measurement of two-photon excitation cross sections of molecular fluorophores with data from 690 to $1050 \mathrm{~nm}$. J Opt Soc Am B 13:481-491

16. Denk W, Svoboda K (1997) Photon upmanship: why multiphoton imaging is more than a gimmick. Neuron 18:351-357

17. Tritthardt J (2002) Entwicklung der Aufnahmeelektronik eines Laser-Scanning-Mikroskops. Fern-Universität-Gesamthochschule Hagen, Hagen/Heidelberg

18. Ernstoff MN, Leupp AM, Little MJ, Peterson HT (1973) Liquid crystal pictorial display. International Electron Devices Meeting. pp. 548-551

19. Siegman AE (1986) Lasers. University Science Books, Sausalito, CA

20. Nathans J (1999) The evolution and physiology of human color vision: insights from molecular genetic studies of visual pigments. Neuron 24:299-312

21. Jacobs GH, Neitz J, Deegan JFd (1991) Retinal receptors in rodents maximally sensitive to ultraviolet light. Nature 353:655-656

22. Sun W, Li N, He S (2002) Large-scale morphological survey of mouse retinal ganglion cells. J Comp Neurol 451:115-126

23. Kelly TL, Munch J (1998) Wavelength dependence of twisted nematic liquid crystal phase modulators. Opt Comm 156: $252-258$

24. Chen E (1993) Refractive indices of the rat retinal layers. Ophthalmic Res 25:65-68

25. Masland RH, Mills JW, Hayden SA (1984) Acetylcholinesynthesizing amacrine cells: identification and selective staining by using radioautography and fluorescent markers. Proc R Soc Lond B Biol Sci 223:79-100

26. Pyle JL, Kavalali ET, Choi S, Tsien RW (1999) Visualization of synaptic activity in hippocampal slices with FM1-43 enabled by fluorescence quenching. Neuron 24:803-808
27. Oesch N, Euler T, Taylor WR (2005) Direction-selective dendritic action potentials in rabbit retina. Neuron 47:739-750

28. Lichtman JW, Wilkinson RS, Rich MM (1985) Multiple innervation of tonic endplates revealed by activity-dependent uptake of fluorescent probes. Nature 314:357-359

29. Ogilvy CS, Borges LF (1988) A quantitative analysis of the retrograde axonal transport of 4 different fluorescent dyes in peripheral sensory and motor neurons and lack of anterograde transport in the corticospinal system. Brain Res 475:244-253

30. Denk W, Detwiler PB (1999) Optical recording of light-evoked calcium signals in the functionally intact retina. Proc Natl Acad Sci USA 96:7035-7040

31. Lewis PR (1955) A theoretical interpretation of spectral sensitivity curves at long wavelengths. J Physiol 130:45-52

32. Stiles WS (1959) Color vision - the approach through incrementthreshold sensitivity. Proc Natl Acad Sci USA 45:100-114

33. Lamb TD (1995) Photoreceptor spectral sensitivities: common shape in the long-wavelength region. Vis Res 35:3083-3091

34. Lyubarsky AL, Daniele LL, Pugh EN Jr (2004) From candelas to photoisomerizations in the mouse eye by rhodopsin bleaching in situ and the light-rearing dependence of the major components of the mouse ERG. Vis Res 44:3235-3251

35. Birge RR (1990) Photophysics and molecular electronic applications of the rhodopsins. Annu Rev Phys Chem 41:683-733

36. Denk W, Piston DW, Webb WW (1995) Two-photon molecular excitation in laser-scanning microscopy. In: Pawley JB (ed) Handbook of biological confocal microscopy. Plenum, New York, pp 445-458

37. Stiles WS, Crawford BH (1933) The luminous efficiency of rays entering the eye pupil at different points. Proc R Soc Lond B 112:428-450

38. Enoch JM (1963) Optical properties of the retinal receptors. J Opt Soc Am A 53:71-85

39. Baylor DA, Fettiplace R (1975) Light path and photon capture in turtle photoreceptors. J Physiol 248:433-464

40. Gray-Keller M, Denk W, Shraiman B, Detwiler PB (1999) Longitudinal spread of second messenger signals in isolated rod outer segments of lizards. J Physiol 519 Pt 3:679-692

41. Boycott BB, Wässle H (1974) The morphological types of ganglion cells of the domestic cat's retina. J Physiol 240:397-419

42. Margolis DJ, Detwiler PB (2007) Different mechanisms generate maintained activity in $\mathrm{ON}$ and $\mathrm{OFF}$ retinal ganglion cells. J Neurosci 27:5994-6005

43. Davenport CM, Detwiler PB, Dacey DM (2007) Functional polarity of dendrites and axons of primate A1 amacrine cells. Vis Neurosci 24:449-457

44. Margolis DJ, Newkirk G, Euler T, Detwiler PB (2008) Functional stability of retinal ganglion cells after degeneration-induced changes in synaptic input. J Neurosci 28:6526-6536 